\title{
Multivariate wavelet Whittle estimation in long-range dependence.
}

\author{
S. Achard ${ }^{1,2}$ and I. Gannaz ${ }^{3}$
}

October 2015

\begin{abstract}
Multivariate processes with long-range dependent properties are found in a large number of applications including finance, geophysics and neuroscience. For real data applications, the correlation between time series is crucial. Usual estimations of correlation can be highly biased due to phase-shifts caused by the differences in the properties of autocorrelation in the processes. To address this issue, we introduce a semiparametric estimation of multivariate long-range dependent processes. The parameters of interest in the model are the vector of the long-range dependence parameters and the long-run covariance matrix, also called functional connectivity in neuroscience. This matrix characterizes coupling between time series. The proposed multivariate wavelet-based Whittle estimation is shown to be consistent for the estimation of both the long-range dependence and the covariance matrix and to encompass both stationary and nonstationary processes. A simulation study and a real data example are presented to illustrate the finite sample behaviour.
\end{abstract}

Keywords: multivariate processes, long memory, fractional integration, semiparametric estimation, covariance matrix, wavelets, neuroscience application, functional connectivity

MSC classification: 60G22, 62M10, 62M15, 62H20, 92C55

\section{Introduction}

The long-range dependence has attracted lots of interest in statistics and in many applications since the seminal paper of Mandelbrot in 1950. First the fractional Brownian motion model was introduced as the unique Gaussian process having stationary increments and self-similarity index $H$ in $(0,1)$ (Mandelbrot and Van Ness, 1968). This model is characterized by one parameter called the Hurst exponent. Since then, several extensions were introduced in order to get more complex modellings that better match real data, such as ARIMA, FD, FIN... We refer to Percival and Walden (2006) and references therein for an overview of long-range dependence models. These models were used in a large scope of applications, for example finance (Gençay et al, 2001) (see also the references in Nielsen and Frederiksen (2005)), internet traffic analysis (Abry and Veitch, 1998),

\footnotetext{
${ }^{1}$ Univ. Grenoble Alpes, GIPSA-Lab, F-38000 Grenoble, France

${ }^{2}$ CNRS, GIPSA-Lab, F-38000 Grenoble, France

${ }^{3}$ Université de Lyon, CNRS UMR 5208, INSA de Lyon, Institut Camille Jordan, France
} 
physical sciences (Papanicolaou and Sølna, 2003; Percival and Walden, 2006), geosciences (Whitcher and Jensen, 2000) and neuroimagery (Maxim et al, 2005).

Nowadays, it is common to record data having multiple sensors, such as neuroimagery (functional Magnetic Resonance Imaging or Electroencephalography). Each sensor records the activity of a specific part of the brain. However, the brain is a complex system with complex interactions between its different parts, so researchers were interested in modelling the sensors as multivariate time series. A similar representation is suited for data acquired in geosciences where, for example, time series correspond to temperatures in several part of the earth, like in Whitcher and Jensen (2000). For these two applications, it has been shown that the univariate time series present longrange dependence behaviour. Several models accounting for long memory features have been proposed. In Didier and Pipiras (2011), the multivariate Brownian motion was defined. The values of interactions as defined by the covariance matrix must be carefully chosen so that the model is identifiable (Coeurjolly et al, 2013). Also, the multivariate extension of fractionally difference models was proposed in Chambers (1995), which includes the multivariate extension of ARFIMA models with an explicit expression of the short memory terms. In a recent paper, Kechagias and Pipiras (2015) highlighted the difficulties to extend the notion of long-range dependence to multivariate time series and proposed specific linear representations of long-range dependence. Concerning multivariate ARFIMA models, Lobato (1997); Sela and Hurvich (2008) studied two different classes of extension depending on the order of fractional integration and ARMA models.

Using these long memory models, a typical statistical issue is to estimate the long memory parameter. This characterizes the long-term dependence of the series, which controls many relevant statistical properties. A very large literature exists in the context of univariate time series. First, parametric approaches were considered (Dahlhaus, 1989; Fox and Taqqu, 1986; Giraitis et al, 1997) which provide fast rates of convergence. However these approaches suffer from inconsistency when the short-term component of the model is misspecified. Semiparametric models were then developed to be robust to model misspecification (Robinson, 1994a,b, 1995a,b), where the spectral density is modelled only near zero frequency. In the frequency domain, two popular estimators among the semiparametric ones are the Geweke-Porter-Hudak introduced by Geweke and PorterHudak (1983) and the local Whittle estimator of Robinson (1995a). Wavelet-based estimators were also studied, and proved to be adequate for studying fractal time series. In Abry and Veitch (1998), the authors developed an estimator using log-regression of the wavelet coefficient variance on the scale index. Moulines et al (2008) derived the asymptotic properties of a wavelet Whittle estimator.

Considering multivariate fractionally integrated processes (Chambers, 1995), the estimation of memory parameters and covariance matrix have been first studied in Robinson (1995b). Then Lobato (1999) proposed a semiparametric two-step estimator. Shimotsu (2007) extended this latter approach including phase-shift consideration. Nielsen (2011) proposed an extension based on Abadir et al (2007)'s extended Fourier transform to estimate long memory parameters for nonstationary time series. In a different approach, Sela and Hurvich (2012) defined an estimator based on the average periodogram for a power law in coherency. All these approaches were developed using Fourier log-periodogram. In comparison, there are few wavelet-based estimators of long-range memory parameters in multivariate settings. Frías et al (2008); Wang and Wang (2014) proposed estimation schemes based on multidimensional wavelet basis. In many real data applications such as geosciences, internet traffic or neurosciences, the number of time series is huge, as the real data example of Section 6 illustrates. The latter works thus do not seem well adapted. 
Achard et al (2008) studied a two-dimensional estimation, based on univariate wavelet basis, which defines estimators using a regression of the cross-covariance between the wavelet coefficients. This approach also appears difficult to generalize to high multidimensional settings. The present work defines a wavelet Whittle estimator for multivariate models. The extension to multivariate processes presents two issues. First, a vector of long memory parameters has to be estimated along with the covariance matrix that is modelling the interactions between the time series. Second, as noted in Robinson (1994b); Shimotsu (2007), the multivariate extension of the fractional integrated model introduces a phase-shift that has to be taken into account in the estimation procedures. The new wavelet-based proposed methodology is shown to be adequate for nonstationary long-range dependence models.

The paper is organised as follows. Section 2 introduces the specific framework of multivariate long memory processes based on the definition of the spectral density matrix. The multivariate wavelet Whittle estimators of both the long memory parameters and the covariance matrix are defined in Section 3. The properties of this new estimation scheme are derived in Section 4 where consistency of both estimations are established. Finally, Section 5 presents a simulation study which illustrates that the wavelet Whittle estimators have comparable performances to the Fourier-based ones. In addition, our method provides a very flexible approach to handle both stationary and nonstationary processes. Section 6 deals with a real data application in neuroscience.

\section{The semiparametric multivariate long-memory framework}

Let $\mathbf{X}=\left\{X_{\ell}(k), k \in \mathbb{Z}, \ell=1, \ldots, p\right\}$ be a multivariate stochastic process. Each process $X_{\ell}$ is not necessarily stationary. Denote by $\Delta X_{\ell}$ the first order difference, $\left(\Delta X_{\ell}\right)(k)=X_{\ell}(k)-X_{\ell}(k-1)$, and by $\Delta^{D} X_{\ell}$ the $D$-th order difference. For every component $X_{\ell}$, there exists $D_{\ell} \in \mathbb{N}$ such that the $D_{\ell}$-th order difference $\Delta^{D_{\ell}} X_{\ell}$ is covariance stationary. Following Achard et al (2008); Chambers (1995); Moulines et al (2007), we consider a long memory process $\mathbf{X}$ with memory parameters $\mathbf{d}=\left(d_{1}, d_{2}, \ldots, d_{p}\right)$. For any $\mathbf{D}>\mathbf{d}-1 / 2$, we suppose that the multivariate process $\mathbf{Z}=\operatorname{diag}\left(\Delta^{D_{\ell}}, \ell=1, \ldots, p\right) \mathbf{X}$ is covariance stationary with a spectral density matrix given by

$$
\text { for all }(\ell, m), f_{\ell, m}^{\left(D_{\ell}, D_{m}\right)}(\lambda)=\frac{1}{2 \pi} \Omega_{\ell, m}\left(1-e^{-i \lambda}\right)^{-d_{\ell}^{s}}\left(1-e^{i \lambda}\right)^{-d_{m}^{s}} f_{\ell, m}^{S}(\lambda), \quad \lambda \in[-\pi, \pi],
$$

where the long memory parameters are given by $d_{m}^{S}=d_{m}-D_{m}$ for all $m$. The functions $f_{\ell, m}^{S}(\cdot)$ correspond to the short memory behaviour of the process. The generalized cross-spectral density of processes $X_{\ell}$ and $X_{m}$ can be written as

$$
f_{\ell, m}(\lambda)=\frac{1}{2 \pi} \Omega_{\ell, m}\left(1-e^{-i \lambda}\right)^{-d_{\ell}}\left(1-e^{i \lambda}\right)^{-d_{m}} f_{\ell, m}^{S}(\lambda), \quad \lambda \in[-\pi, \pi] .
$$

As it will be explained in Section 2.1, this model corresponds to a subclass of multivariate longrange dependent time series. In a general case, an additional multiplicative term of the form $e^{i \varphi}$ is required, see e.g. Kechagias and Pipiras (2015); Sela and Hurvich (2012).

In the case of a multivariate setting, the spectral density of the multivariate process $\mathbf{X}$ is thus,

$$
f(\lambda)=\mathbf{\Omega} \circ\left(\boldsymbol{\Lambda}^{0}(\mathbf{d}) f^{\mathcal{S}}(\lambda) \Lambda^{0}(\mathbf{d})^{*}\right), \quad \lambda \in[-\pi, \pi], \quad \text { with } \boldsymbol{\Lambda}^{0}(\mathbf{d})=\operatorname{diag}\left(\left(1-e^{-i \lambda}\right)^{-\mathbf{d}}\right)
$$


where $\mathbf{d}=\mathbf{D}+\mathbf{d}^{s}$. The exponent $*$ is the conjugate operator and $\circ$ denotes the Hadamard product. The matrix $\Omega$ is supposed real symmetric positive definite.

In this semiparametric framework, the spectral density $f^{\mathcal{S}}(\cdot)$ corresponds to the short-memory behaviour and the matrix $\Omega$ is called fractal connectivity by Achard et al (2008) or long-run covariance matrix by Robinson (2005). Similarly to Moulines et al (2007), we assume that $f^{\mathcal{S}}(\cdot) \in \mathcal{H}(\beta, L)$ with $0<\beta \leqslant 2$ and $0<L$. The space $\mathcal{H}(\beta, L)$ is defined as the class of non-negative symmetric functions $\mathbf{g}(\cdot)$ on $[\pi, \pi]$ such that $g_{\ell, m}(0)=1$ for all $(\ell, m) \in\{1, \ldots, p\}^{2}$ and that for all $\lambda \in(-\pi, \pi)$, $\max \left\{|\mathbf{g}(\lambda)-1|,(\ell, m) \in\{1, \ldots, p\}^{2}\right\}_{\infty} \leqslant L|\lambda|^{\beta}$. The assumption $f_{\ell, m}^{S}(0)=1$ for all $(\ell, m)$ is necessary for $\Omega$ to be identifiable in model (1).

The spectral density specifies that the two processes $X_{\ell}$ and $X_{m}$ have long-memory parameters respectively $d_{\ell}$ and $d_{m}$. Parameters with absolute value greater that $1 / 2$ are allowed, covering nonstationary time series (in this case $D_{\ell}, D_{m} \geqslant 1$ ). If orders are different, the estimation of the memory parameters is still available but some bias issues occur for the estimation of the underlying covariance $\Omega$, which is detailled in Section 3 .

In order to derive semiparametric estimations of the memory parameters and the matrix $\Omega$, the term inside the matrix $\Lambda^{0}(\mathbf{d})$ can be simplified using the equality $1-e^{-i \lambda}=2 \sin (\lambda / 2) e^{i(\pi-\lambda) / 2}$. Consequently, when $\lambda$ tends to 0 , the spectral density matrix is approximated at first order by

$$
f(\lambda) \sim \widetilde{\Lambda}(\mathbf{d}) \mathbf{\Omega} \widetilde{\boldsymbol{\Lambda}}(\mathbf{d})^{*}, \quad \text { when } \lambda \rightarrow 0, \quad \text { with } \tilde{\boldsymbol{\Lambda}}(\mathbf{d})=\operatorname{diag}\left(|\lambda|^{-\mathbf{d}} e^{-i \operatorname{sign}(\lambda) \pi \mathbf{d} / 2}\right) .
$$

Here and subsequently $\sim$ means that the ratio left- and right-hand side converges to one.

A similar approximation has been carried out in Lobato (1997) or Phillips and Shimotsu (2004), while Shimotsu (2007) derived a second order approximation. Lobato (1999) used $\widetilde{\Lambda}(\mathbf{d})=$ $\operatorname{diag}\left(|\lambda|^{-\mathbf{d}}\right)$ as an approximation of $f(\cdot)$. Whereas Shimotsu (2007) chose to approximate $f(\cdot)$

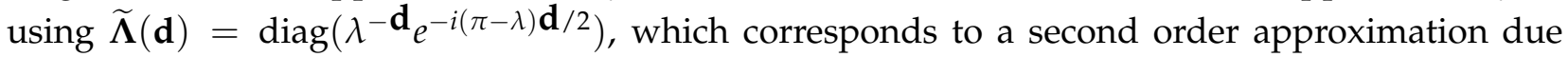
to the remaining term $\lambda$ in the exponential. As mentioned by Shimotsu (2007), intriguingly, the two defined estimators of long memory parameters are consistent, but only for the estimation of d. The estimation of the covariance matrix is affected by the choice of $\widetilde{\Lambda}(\mathbf{d})$. In Section 3 , we introduce our estimators using approximation (2), corresponding to a trade-off between Lobato (1999) and Shimotsu (2007). The resulting estimator for $\mathbf{d}$ is equivalent to the one defined in Lobato (1999). However a specific correction for the estimation of the covariance matrix overcomes the bias caused by the presence of a phase-shift through the complex exponential term. This point has also been raised in the context of detecting cointegration, when the cross-spectral density presents an additional phase parameter comparing to the case studied in this paper.

\subsection{Examples of processes}

This section provides some examples of processes which satisfy our semiparametric modelling.

The matrix $\Omega$ has been defined via the spectral representation of the process, the link between $\Omega$ and the covariance of the multivariate process in the temporal space is detailed hereafter. Let 
$\overline{\mathbf{X}}=\frac{1}{N} \sum_{t=1}^{N} \mathbf{X}(t)$ be the empirical mean of the process. If the cross-spectral density is defined and continuous at the frequency $\lambda=0$, Fejer's theorem states that $n^{1 / 2} \overline{\mathbf{X}}$ converges in distribution to a zero-mean Gaussian distribution with a covariance matrix equals to $2 \pi f(0)$. When the crossspectral density satisfies an approximation (1), Robinson (2005) indicates that

$$
\mathbf{D}_{n} \mathbb{E}\left(\overline{\mathbf{X X}}^{T}\right) \mathbf{D}_{n} \underset{n \rightarrow \infty}{\longrightarrow} 2 \pi \Omega \circ \mathbf{Q}(\mathbf{d})
$$

where $\mathbf{D}_{n}=\operatorname{diag}\left(n^{1 / 2-\mathbf{d}}\right)$ and $Q_{\ell, m}(\mathbf{d})=\frac{\sin \left(\pi d_{\ell}\right)+\sin \left(\pi d_{m}\right)}{\Gamma\left(d_{\ell}+d_{m}+2\right) \sin \left(\pi\left(d_{\ell}+d_{m}\right)\right)}$. The exponent $T$ denotes the transpose operator.

\subsubsection{Causal linear representations of Kechagias and Pipiras (2014)}

Kechagias and Pipiras (2015) define Long-Range Dependence (LRD) with a more general setting for the phase. Let $\mathbf{X}$ be a $p$-multivariate time series. We suppose that $\mathbf{X}$ is second-order stationary and that it admits a spectral density. The time series $\mathbf{X}$ is long-range dependent in the sense of Kechagias and Pipiras (2015) if its spectral density $f(\cdot)$ satisfies:

$$
f(\lambda)=\frac{1}{2 \pi} \operatorname{diag}\left(|\lambda|^{-\mathbf{d}}\right) \mathbf{G}(\lambda) \operatorname{diag}\left(|\lambda|^{-\mathbf{d}}\right)
$$

with $\mathbf{d}=\left(\begin{array}{lll}d_{1} & \ldots & d_{p}\end{array}\right) \in(0,1 / 2)^{p}$ and $\mathbf{G}(\cdot)$ a $\mathbb{C}^{p \times p}$-valued Hermitian non negative definite matrix function satisfying

$$
\mathbf{G}(\lambda) \sim_{\lambda \rightarrow 0^{+}} \mathbf{G}=\left(\Omega_{\ell, m} e^{i \varphi_{\ell, m}}\right)_{\ell, m=1, \ldots, p}
$$

with $\Omega_{\ell, m} \in \mathbb{R}, \ell, m=1, \ldots, p$ and $\varphi_{\ell, m} \in(-\pi, \pi]$. The phases $\varphi_{\ell, m}$ measure the dissymmetry of the process $\mathbf{X}$ at large lags. Indeed the specificity of multivariate time series is that the autocovariance function may no longer be symmetric compared with the univariate framework (i.e. $\gamma(-h)=\gamma(h)^{T}$ may not be equal to $\gamma(h)$ ). When the process is time reversible, the autocovariance function is symmetric. Time reversible series will satisfy $\varphi_{\ell, m}=0$ for all $\ell, m=1, \ldots, p$. We refer to proposition 2.1 of Kechagias and Pipiras (2015) that gives some highlights on the phase parameters. In Proposition 3.1 Kechagias and Pipiras (2015) give examples of LRD linear time series where any combinations of $(\mathbf{d}, \mathbf{G})$ can be chosen.

Many results of the present work can be generalized to LRD processes of Kechagias and Pipiras (2015). Yet as our goal is to recover the matrix $\Omega$, an assumption on the form of the phase $\varphi$ is necessary (since we consider a real filter which deletes the imaginary part). The most widely used definition of phase is $\varphi_{\ell, m}=-\frac{\pi}{2}\left(d_{\ell}-d_{m}\right)$ which includes a large scope of models, see section 2.1.2.

Such a definition of phase is verified, for example, using a causal representation of processes described in Kechagias and Pipiras (2015). Let $\left\{\varepsilon_{k}\right\}_{k \in \mathbb{Z}}$ be a $\mathbb{R}^{p}$-valued white noise, satisfying $\mathbb{E}\left[\boldsymbol{\varepsilon}_{k}\right]=0$ and $\mathbb{E}\left[\boldsymbol{\varepsilon}_{k} \boldsymbol{\varepsilon}_{k}^{T}\right]=\mathbf{I}_{p}$. Let also $\left\{\mathbf{B}_{k}=\left(B_{\ell, m, k}\right)_{\ell, m=1, \ldots, p}\right\}_{k \in \mathbb{N}}$ be a sequence of real-valued matrices such that $B_{\ell, m, k}=L_{\ell, m}(k) k^{d_{a}-1}, k \in \mathbb{N}$, where $d_{\ell} \in(0,1 / 2)$ and $\mathbf{L}(k), k=1, \ldots, p$, is an $\mathbb{R}^{p \times p}$-valued function satisfying $\mathbf{L}(k) \sim_{k \rightarrow+\infty} \mathbf{A}$ for some $\mathbb{R}^{p \times p}$-valued matrix $\mathbf{A}$. We define the time series $\mathbf{X}$ given by the causal linear representation

$$
\mathbf{X}(k)=\sum_{j=0}^{+\infty} \mathbf{B}_{j} \varepsilon_{k-j}
$$


Corollary 4.1 of Kechagias and Pipiras (2015) states that the process $\mathbf{X}$ is LRD with

$$
\begin{aligned}
\Omega_{\ell, m} & =\frac{\Gamma\left(d_{\ell}\right) \Gamma\left(d_{m}\right)}{2 \pi}\left(\mathbf{A A}^{*}\right)_{\ell, m}, \\
\varphi_{\ell, m} & =-\frac{\pi}{2}\left(d_{\ell}-d_{m}\right) .
\end{aligned}
$$

Such causal linear representations thus satisfies (2). An example of such a representation is the multivariate $\operatorname{ARFIMA}(0, \mathbf{d}, 0)$ model presented in next subsection.

\subsubsection{Multivariate ARFIMA of Lobato (1997)}

The composition of linear filters does not commute in the multivariate case. Consequently there are multiple extensions of univariate ARFIMA to the multivariate framework. We detail in this section the multivariate ARFIMA models of Lobato (1997).

Let $\mathbf{u}$ be a $p$-dimensional white noise with $\mathbb{E}\left[\mathbf{u}(t) \mid \mathcal{F}_{t-1}\right]=0$ and $\mathbb{E}\left[\mathbf{u}(t) \mathbf{u}(t)^{T} \mid \mathcal{F}_{t-1}\right]=\boldsymbol{\Sigma}$, where $\mathcal{F}_{t-1}$ is the $\sigma$-field generated by $\{\mathbf{u}(s), s<t\}$, and $\Sigma$ is a positive definite matrix. The spectral density of $\mathbf{u}$ satisfies $f_{u}(\lambda)=\Sigma /(2 \pi)$.

Let $\left(\mathbf{A}_{k}\right)_{k \in \mathbb{N}}$ be a sequence of $\mathbb{R}^{p \times p}$-valued matrices with $\mathbf{A}_{0}$ the identity matrix and $\sum_{k=0}^{\infty}\left\|\mathbf{A}_{k}\right\|^{2}<$ $\infty$. Let $\mathbf{A}(\cdot)$ be the discrete Fourier transform of the sequence, $\mathbf{A}(\lambda)=\sum_{k=0}^{\infty} \mathbf{A}_{k} e^{i k \lambda}$. We assume that all the roots of $|\mathbf{A}(\mathbb{L})|$ are outside the closed unit circle.

Lobato (1997) defines two multivariate ARFIMA models which both satisfy approximation (2).

Model A. Let $\mathbf{X}$ be defined by $\mathbf{A}(\mathbb{L}) \operatorname{diag}(\mathbb{1}-\mathbb{L})^{\mathbf{d}} \mathbf{X}(t)=\mathbf{B}(\mathbb{L}) \mathbf{u}(t)$. The spectral density satisfies

$$
f_{\ell, m}(\lambda) \sim_{\lambda \rightarrow 0^{+}} \frac{1}{2 \pi} \Omega_{\ell, m} e^{-i \pi / 2\left(d_{\ell}-d_{m}\right)} \lambda^{-\left(d_{\ell}+d_{m}\right)}
$$

with $\Omega=\mathbf{A}(1)^{-1} \mathbf{B}(1) \boldsymbol{\Sigma} \mathbf{B}(1)^{T} \mathbf{A}(1)^{T^{-1}}$. It is straightforward that Model A statifies approximation (2).

Model B. Let $\mathbf{X}$ be defined by $\operatorname{diag}(\mathbb{1}-\mathbb{L})^{\mathbf{d}} \mathbf{A}(\mathbb{L}) \mathbf{X}(t)=\mathbf{B}(\mathbb{L}) \mathbf{u}(t)$. The spectral density satisfies

$$
f_{\ell, m}(\lambda) \sim_{\lambda \rightarrow 0^{+}} \frac{1}{2 \pi} \sum_{a, b} \beta_{a, b} \alpha_{\ell, a} \alpha_{m, b} e^{-i \pi / 2\left(d_{a}-d_{b}\right)} \lambda^{-\left(d_{a}+d_{b}\right)}
$$

with $\alpha_{\ell, m}=\left(\mathbf{A}(1)^{-1}\right)_{\ell, m}$ and $\beta_{\ell, m}=\left(\mathbf{B}(1) \mathbf{\Sigma} \mathbf{B}(1)^{T}\right)_{\ell, m}$. In Model B the spectral density is equivalent around the zero frequency to the term in $(a, b)=\operatorname{argmax}\left\{\left|d_{a}+d_{b}\right|, \beta_{a, b} \alpha_{\ell, a} \alpha_{m, b} \neq\right.$ $0\}$. It gives a more general setting $f_{\ell, m}(\lambda) \sim_{\lambda \rightarrow 0^{+}} G_{\ell, m} \lambda^{-d_{\ell, m}}$ with $\left|d_{\ell, m}\right| \leqslant\left(d_{\ell}+d_{m}\right) / 2$. (This model is studied more extensively in Sela and Hurvich (2012), where the authors propose an estimator for $d_{1,2}$ in a bivariate framework.) Setting $\Omega_{\ell, m}=0$ if $\left|d_{\ell, m}\right|<\left(d_{\ell}+d_{m}\right) / 2$ equation (2) holds. This means that if there is cointegration, the corresponding long-run covariance value is set to zero. 
In particular, Model A and Model B of Lobato (1997) include FIVAR and VARFI models of Sela and Hurvich (2008). These multivariate ARFIMA models admit a causal linear representation. They include short-range dependence behaviour, through the terms $A(\mathbb{L})$ and $B(\mathbb{L})$. When these terms are equal to identity, we obtain an $\operatorname{ARFIMA}(0, \mathbf{d}, 0)$ satisfying the causal linear representation given in subsection 2.1.1. This (causal) multivariate $\operatorname{ARFIMA}(0, \mathbf{d}, 0)$ is a subclass of Kechagias and Pipiras (2015)'s (possibly non causal) definition.

Remark. In the univariate setting, when $A(\cdot)$ and $B(\cdot)$ have no common zeros, Kokoszka and Taqqu (1995) establish that the time series $X$ admits a linear representation $X(k)=\sum_{j=0}^{+\infty} C_{j} \varepsilon_{k-j}$ with $C_{j}=\frac{B(1)}{A(1) \Gamma(d)} j^{d-1}+O\left(j^{-1}\right)$ when $j$ goes to infinity. The terms of the linear representation thus satisfy approximately the condition of Kechagias and Pipiras (2015). The extension to multivariate setting would yield information about the link between Lobato (1997)'s models and Kechagias and Pipiras (2015)'s condition on causal representations. However, it has not been explored in this paper since we will not use this fact in any essential way.

\section{Multivariate wavelet Whittle estimation}

This section first defines the wavelet transform of the processes and then gives some results on the cross behaviour of the wavelet coefficients. The main point is the presence of a phase-shift caused by the differences in the long-memory parameters. Finally the proposed estimation scheme is derived, defining simultaneous estimators of the long-memory parameters and of the long-run covariance, which takes into account the phase-shift (based on the first order approximation (2)).

\subsection{The wavelet analysis}

Let $(\phi(\cdot), \psi(\cdot))$ be respectively a father and a mother wavelets. Their Fourier transforms are given by $\widehat{\phi}(\lambda)=\int_{-\infty}^{\infty} \phi(t) e^{-i \lambda t} d t$ and $\widehat{\psi}(\lambda)=\int_{-\infty}^{\infty} \psi(t) e^{-i \lambda t} d t$.

At a given resolution $j \geqslant 0$, for $k \in \mathbb{Z}$, we define the dilated and translated functions $\phi_{j, k}(\cdot)=$ $2^{-j / 2} \phi\left(2^{-j} \cdot-k\right)$ and $\psi_{j, k}(\cdot)=2^{-j / 2} \psi\left(2^{-j} \cdot-k\right)$. Thoughout the paper, we adopt the same convention as in Moulines et al (2007) and Moulines et al (2008), that is large values of the scale index $j$ correspond to coarse scales (low frequencies).

Let $\widetilde{\mathbf{X}}(t)=\sum_{k \in \mathbb{Z}} \mathbf{X}(k) \phi(t-k)$. The wavelet coefficients of the process $\mathbf{X}$ are defined by

$$
\mathbf{W}_{j, k}=\int_{\mathbb{R}} \widetilde{\mathbf{X}}(t) \psi_{j, k}(t) d t \quad j \geqslant 0, k \in \mathbb{Z}
$$

For given $j \geqslant 0$ and $k \in \mathbb{Z}, \mathbf{W}_{j, k}$ is a $p$-dimensional vector $\mathbf{W}_{j k}=\left(\begin{array}{llll}W_{j, k}(1) & W_{j, k}(2) \quad \ldots \quad W_{j, k}(p)\end{array}\right)$, where $W_{j, k}(\ell)=\int_{\mathbb{R}} \widetilde{X}_{\ell}(t) \psi_{j, k}(t) d t$.

The regularity conditions on the wavelet transform are expressed in the following assumptions. They will be needed throughout the paper. 
(W1) The functions $\phi(\cdot)$ and $\psi(\cdot)$ are integrable, have compact supports, $\int_{\mathbb{R}} \phi(t) d t=1$ and $\int \psi^{2}(t) d t=1 ;$

(W2) There exists $\alpha>1$ such that $\sup _{\lambda \in \mathbb{R}}|\widehat{\psi}(\lambda)|(1+|\lambda|)^{\alpha}<\infty$, i.e. the wavelet is $\alpha$-regular;

(W3) The mother wavelet $\psi(\cdot)$ has $M>1$ vanishing moments.

(W4) The function $\sum_{k \in \mathbb{Z}} k^{\ell} \phi(\cdot-k)$ is polynomial with degree $\ell$ for all $\ell=1, \ldots, M-1$.

(W5) For all $i=1, \ldots, p,(1+\beta) / 2-\alpha<d_{i} \leqslant M$.

These conditions are not restrictive, and many standard wavelet basis satisfy them. Among them, Daubechies wavelets are compactly supported wavelets parametrized by the number of vanishing moments $M$. They are $\alpha$-regular with $\alpha$ an increasing function of $M$ going to infinity (see Daubechies (1992)). Assumptions (W1)-(W5) will hold for Daubechies wavelet basis with sufficiently large $M$.

Remark. The couple of functions $(\phi(\cdot), \psi(\cdot))$ can be associated with a multiresolution analysis, but this condition is not necessary. Similarly, the orthogonality of the family $\left\{\psi_{j, k}(\cdot)\right\}$ is not required. See Moulines et al (2007), Section 3.

Under assumption (W3), the wavelet transform performs an implicit differentiation of order $M$. Thus it is possible to apply it on nonstationary processes. In Fourier analysis, tapering procedures are necessary to consider directly nonstationary frameworks, see e.g. Velasco and Robinson (2000) and references therein. Some recent works propose a procedure that differentiates the data before tapering (Hurvich and Chen (2000) and references therein). Another extension of Fourier to nonstationary frameworks has been proposed by Abadir et al (2007) and applied by Nielsen (2011) in multivariate analysis.

In practice, a finite number of realisation of the process $\mathbf{X}$, say $\mathbf{X}(1), \ldots \mathbf{X}(N)$, is observed. Since the wavelets have a compact support only a finite number $n_{j}$ of coefficients are non null at each scale $j$. Suppose without loss of generality that the support of $\psi(\cdot)$ is included in $\left[0, T_{\psi}\right]$ with $T_{\psi} \geqslant 1$. For every $j \geqslant 0$, define

$$
n_{j}:=\max \left(0,2^{-j}\left(N-T_{\psi}+1\right)\right) .
$$

Then for every $k<0$ and $k>n_{j}$, the coefficients $\mathbf{W}_{j, k}$ are set to zero because all the observations are not available. In the following, $n=\sum_{j=j_{0}}^{j_{1}} n_{j}$ denotes the total number of non-zero coefficients used for estimation.

\subsection{Spectral approximation of wavelet coefficients}

Let us first recall some results of Moulines et al (2007) for the wavelet transform of a univariate process. Let $W_{j, k}$ denote the wavelet coefficient of a unidimensional process $X$, with spectral density $f(\lambda)=\left|1-e^{i \lambda}\right|^{-2 d_{0}} f^{S}(\lambda)$, where $d_{0} \in \mathbb{R}$ (note that $d_{0}$ can be outside of the interval $[-1 / 2,1 / 2]$ ). Moulines et al (2007) state that under assumptions (W1)-(W5), the wavelet coefficients process $\left(W_{j, k}\right)_{k \in \mathbb{Z}}$ is covariance stationary for any given $j \geqslant 0$. However they also stress that the betweenscale coefficients are not decorrelated. It is shown that the wavelet coefficients are decorrelated 
when the wavelet bases is orthonormal and $d_{0}=0$ but it is not valid in general settings. Many proposition of estimators of long-memory can be found in Abry and Veitch (1998); Gonzaga and Hauser (2011); Jensen (1999); Wornell and Oppenheim (1992), among others. These works assume that the wavelet coefficients are decorrelated. We follow Bardet et al (2000) or Moulines et al (2008) in taking into account the within and between scales behaviour.

Let $j \geqslant 0$ and $j^{\prime}=j-u \leqslant j$ be two given scales. Following Moulines et al (2007), the between-scale process is defined as the sequence $\left\{W_{j, k}, W_{j-u, 2^{u} k+\tau}, \tau=0, \ldots 2^{u}-1\right\}_{k \in \mathbb{Z}}$. Let $\mathbf{D}_{j, u}\left(\cdot ; d_{0}\right)$ be the crossspectral density between $\left\{W_{j, k}\right\}_{k \in \mathbb{Z}}$ and $\left\{W_{j-u, 2^{u} k+\tau}, \tau=0, \ldots 2^{u}-1\right\}_{k \in \mathbb{Z}}$. For any $\lambda \in(-\pi, \pi)$, $\mathbf{D}_{j, u}\left(\lambda ; d_{0}\right)$ is a $2^{u}$-dimensional vector. Theorem 1 in Moulines et al (2007) establishes that under assumptions (W1)-(W5) there exists a positive constant $C$ such that for all $\lambda \in(-\pi, \pi)$,

$$
\left|\mathbf{D}_{j, u}\left(\lambda ; d_{0}\right)-2^{2 j d_{0}} \mathbf{D}_{\infty, u}\left(\lambda ; d_{0}\right)\right| \leqslant C 2^{j\left(2 d_{0}-\beta\right)},
$$

where

$$
\mathbf{D}_{\infty, u}\left(\lambda ; d_{0}\right):=\sum_{t \in \mathbb{Z}}|\lambda+2 t \pi|^{-2 d_{0}} \widehat{\psi}^{*}(\lambda+2 t \pi) 2^{-u / 2} \widehat{\psi}\left(2^{-u}(\lambda+2 t \pi)\right) \mathbf{e}_{u}(\lambda+2 t \pi)
$$

with $\mathbf{e}_{u}(\xi)=\left(\begin{array}{llll}1 & e^{-i 2^{-u}} \xi & \ldots & e^{-i 2^{-u}\left(2^{u}-1\right)} \xi\end{array}\right)^{T}$.

The key point of our estimation is the extension of results obtained by Moulines et al (2007) to the multivariate framework. Due to the complexity of the multivariate setting, we choose not to characterize the behaviour of the wavelet coefficients in terms of cross-spectral densities.

First, in order to extend the results of Moulines et al (2007) to a multivariate framework, the covariance behaviour of $\mathbf{W}_{j, k}$ for given $(j, k)$ is derived. Let $\theta_{\ell, m}(j)$ denote the wavelet covariance at scale $j$ between processes $X_{\ell}$ and $X_{m}, \theta_{\ell, m}(j)=\operatorname{Cov}\left(W_{j, k}(\ell), W_{j, k}(m)\right)$ for any position $k$. Using the spectral density representation, $\theta_{\ell, m}(j)$ satisfies

$$
\theta_{\ell, m}(j)=\int_{-\pi}^{\pi}\left(1-e^{-i \lambda}\right)^{-d_{\ell}}\left(1-e^{i \lambda}\right)^{-d_{m}} \Omega_{\ell, m} f_{\ell, m}^{S}(\lambda)\left|\mathbb{H}_{j}(\lambda)\right|^{2} d \lambda
$$

where $\mathbb{H}_{j}$ is the gain function of the wavelet filter.

The following proposition establishes a second order approximation of the spectral density in a neighbourhood of zero, such as the one derived in Shimotsu (2007).

Proposition 1. Let assumptions (W1)-(W5) hold. Let $j \geqslant 0, \ell, m=1, \ldots, p$. Let $K_{j}$ be defined by

$$
K_{j}\left(d_{\ell}, d_{m}\right)=\int_{-\infty}^{\infty}|\lambda|^{-\left(d_{\ell}+d_{m}\right)} \cos \left(2^{-j} \lambda\left(d_{\ell}-d_{m}\right) / 2\right)|\widehat{\psi}(\lambda)|^{2} d \lambda .
$$

Then there exists a constant $C_{0}$ depending on $\beta, \min _{i} d_{i}, \max _{i} d_{i}, \Omega, \phi(\cdot)$ and $\psi(\cdot)$ such that

$$
\left|\theta_{\ell, m}(j)-\Omega_{\ell, m} 2^{j\left(d_{\ell}+d_{m}\right)} \cos \left(\pi\left(d_{\ell}-d_{m}\right) / 2\right) K_{j}\left(d_{\ell}+d_{m}\right)\right| \leqslant C_{0} L 2^{\left(d_{\ell}+d_{m}-\beta\right) j} .
$$

Note that the second order approximation of the spectral density depends on $j$ also through the function $K_{j}$. The following proposition is deriving a first order approximation so that its logarithm is linear in $j$. 
Proposition 2. Let $K(\cdot)$ be defined by

$$
K(\delta)=\int_{-\infty}^{\infty}|\lambda|^{-\delta}|\widehat{\psi}(\lambda)|^{2} d \lambda, \quad \delta \in(-\alpha, M) .
$$

Under assumptions (W1)-(W5), there exists a constant $C$ depending on $\beta, \min _{i} d_{i}, \max _{i} d_{i}, \Omega, \phi(\cdot)$ and $\psi(\cdot)$ such that, for all $j \geqslant 0$, for all $\ell, m=1, \ldots, p$,

$$
\left|\theta_{\ell, m}(j)-\Omega_{\ell, m} 2^{j\left(d_{\ell}+d_{m}\right)} \cos \left(\pi\left(d_{\ell}-d_{m}\right) / 2\right) K\left(d_{\ell}+d_{m}\right)\right| \leqslant C L 2^{\left(d_{\ell}+d_{m}-\beta\right) j} .
$$

Observe that the approximation (5) shows that the difficulty with the case of multivariate longmemory processes is the appearance of a phase-shift that has to be taken into account for the estimation of the covariance $\Omega$. Indeed, $\theta_{\ell, m}(j)$ is proved to be close to a term proportional to $\cos \left(\pi\left(d_{\ell}-d_{m}\right) / 2\right)$. Then, if $d_{\ell} \in[-1 / 2,1 / 2]$ and $d_{m}=2 k+1+d_{\ell}^{S}$ with $k \in \mathbb{N}$, Proposition 2 implies that for all $j, \theta_{\ell, m}(j)$ is negligible, meaning that the covariance of the wavelet coefficients is close to zero. Consequently, using the covariance of the wavelet coefficients does not allow to estimate the matrix $\Omega$ accurately. This example corresponds to a covariance-stationary process $X_{\ell}$ and a process $X_{m}$ such that $\Delta X_{m}$ is covariance stationary, both with the same long-memory parameter $d_{\ell}$. We show in what follows that the consistency of the long memory parameters is not affected by bias in the estimation of $\Omega$.

The covariance behaviour for the between scale process is derived in the following proposition.

Proposition 3. For all $j \geqslant 0, u \geqslant 0$ and $\lambda \in(-\pi, \pi)$, we define

$$
\begin{aligned}
D_{u ; \tau}^{(j)}\left(\lambda ; f_{\ell, m}(\cdot)\right) & =\sum_{t \in \mathbb{Z}} f_{\ell, m}\left(2^{-j}(\lambda+2 t \pi)\right) 2^{-j} \mathbb{H}_{j}\left(2^{-j}(\lambda+2 t \pi)\right) \mathbb{H}_{j-u}^{*}\left(2^{-j}(\lambda+2 t \pi)\right) e^{-i 2^{-u} \tau(\lambda+2 t \pi)} \\
\widetilde{D}_{u, \tau}(\lambda ; \delta) & =\sum_{t \in \mathbb{Z}}|\lambda+2 t \pi|^{-\delta} \widehat{\psi}^{*}(\lambda+2 t \pi) 2^{-u / 2} \widehat{\psi}\left(2^{-u}(\lambda+2 t \pi)\right) e^{-i 2^{-u} \tau(\lambda+2 t \pi)}
\end{aligned}
$$

and $K_{u, \tau}(v ; \delta)=\int_{-\pi}^{\pi} \widetilde{D}_{u, \tau}(\lambda ; \delta) e^{i \lambda v} d \lambda$.

Then for all $j \geqslant 0$, for all $u, v \geqslant 0, \tau=0, \ldots, 2^{u}-1$,

$$
\operatorname{Cov}\left(W_{j, k}(\ell), W_{j-u, 2^{-u} k^{\prime}+\tau}(m)\right)=\int_{-\pi}^{\pi} D_{u ; \tau}^{(j)}(\lambda ;(\ell, m)) e^{i \lambda\left(k-k^{\prime}\right)} d \lambda .
$$

Under assumptions (W1)-(W5), there exists a constant $C$ depending on $\beta, \min _{i} d_{i}, \max _{i} d_{i}, \Omega, \phi(\cdot)$ and $\psi(\cdot)$ such that, for all $j \geqslant 0$, for all $u, v \geqslant 0, \tau=0, \ldots, 2^{u}-1$, for all $\lambda \in(-\pi, \pi)$,

$$
\left|D_{u ; \tau}^{(j)}\left(\lambda ; f_{\ell, m}(\cdot)\right)-\Omega_{\ell, m} 2^{j\left(d_{\ell}+d_{m}\right)} \cos \left(\pi\left(d_{\ell}-d_{m}\right) / 2\right) \widetilde{D}_{u, \tau}\left(\lambda ; d_{\ell}+d_{m}\right)\right| \leqslant C L 2^{\left(d_{\ell}+d_{m}-\beta\right) j} .
$$

and

$$
\begin{aligned}
\mid \operatorname{Cov}\left[W_{j, k}(\ell) W_{j-u, 2^{-u} k^{\prime}+\tau}(m)\right]-\Omega_{\ell, m} 2^{j\left(d_{\ell}+d_{m}\right)} \cos \left(\pi\left(d_{\ell}-d_{m}\right) / 2\right) K_{u, \tau}\left(k-k^{\prime} ; d_{\ell}\right. & \left.+d_{m}\right) \mid \\
& \leqslant C L 2^{\left(d_{\ell}+d_{m}-\beta\right) j} .
\end{aligned}
$$

When $u=0$ and $2^{u} k^{\prime}+\tau=k$, the quantity $K_{0,0}\left(0 ; d_{\ell}+d_{m}\right)$ is equal to $\int_{-\infty}^{\infty}|\lambda|^{-\left(d_{\ell}+d_{m}\right)}|\widehat{\psi}(\lambda)|^{2} d \lambda$. Let us remark that $K_{0,0}(0 ; \cdot)$ is equal to the function $K(\cdot)$ defined in Proposition 2. 


\subsection{Wavelet Whittle estimation}

Let $j_{1} \geqslant j_{0} \geqslant 1$ be respectively the maximal and the minimal resolution levels that are used in the estimation procedure. The estimation is based on the vectors of wavelets coefficients $\left\{\mathbf{W}_{j, k}, j_{0} \leqslant j \leqslant j_{1}, k \in \mathbb{Z}\right\}$.

The wavelet Whittle approximation of the negative log-likelihood is denoted by $\mathcal{L}(\cdot)$. The criterion corresponds to the negative log-likelihood of Gaussian vectors $\left(W_{j, k}(\ell)\right)_{j, k, \ell}$. Fox and Taqqu (1986); Hannan (1973) prove that the Whittle approximation is giving satisfactory results for nongaussian processes. In our framework, the wavelet Whittle criterion is defined as,

$$
\mathcal{L}(\mathbf{G}(\mathbf{d}), \mathbf{d})=\frac{1}{n} \sum_{j=j_{0}}^{j_{1}}\left[n_{j} \log \operatorname{det}\left(\boldsymbol{\Lambda}_{j}(\mathbf{d}) \mathbf{G}(\mathbf{d}) \boldsymbol{\Lambda}_{j}(\mathbf{d})\right)+\sum_{k=0}^{n_{j}} \mathbf{W}_{j, k}^{T}\left(\boldsymbol{\Lambda}_{j}(\mathbf{d}) \mathbf{G}(\mathbf{d}) \boldsymbol{\Lambda}_{j}(\mathbf{d})\right)^{-1} \mathbf{W}_{j, k}\right],
$$

where $\boldsymbol{\Lambda}_{j}(\mathbf{d})$ and the matrix $\mathbf{G}(\mathbf{d})$ are obtained with Proposition 2,

$$
\Lambda_{j}(\mathbf{d})=\operatorname{diag}\left(2^{j \mathbf{d}}\right)
$$

and the $(\ell, m)$-th element of the matrix $\mathbf{G}(\mathbf{d})$ is $G_{\ell, m}(\mathbf{d})=\Omega_{\ell, m} K\left(d_{\ell}+d_{m}\right) \cos \left(\pi\left(d_{\ell}-d_{m}\right) / 2\right)$.

For each $j \geqslant 0$, the quantity $\sum_{k} \mathbf{W}_{j, k}^{T}\left(\boldsymbol{\Lambda}_{j}(\mathbf{d}) \mathbf{G}(\mathbf{d}) \boldsymbol{\Lambda}_{j}(\mathbf{d})\right)^{-1} \mathbf{W}_{j, k}$ has a dimension equal to 1 and is equal to its trace. Thus,

$$
\mathcal{L}(\mathbf{G}(\mathbf{d}), \mathbf{d})=\frac{1}{n} \sum_{j=j_{0}}^{j_{1}}\left[n_{j} \log \operatorname{det}\left(\boldsymbol{\Lambda}_{j}(\mathbf{d}) \mathbf{G}(\mathbf{d}) \boldsymbol{\Lambda}_{j}(\mathbf{d})\right)+\operatorname{trace}\left(\left(\boldsymbol{\Lambda}_{j}(\mathbf{d}) \mathbf{G}(\mathbf{d}) \boldsymbol{\Lambda}_{j}(\mathbf{d})\right)^{-1} \mathbf{I}(j)\right)\right],
$$

where $\mathbf{I}(j)=\sum_{k=0}^{n_{j}} \mathbf{W}_{j, k} \mathbf{W}_{j, k}^{T}$. Note that this expression is very similar to the multivariate Fourier Whittle estimator of Shimotsu (2007). Here we replace the periodogram by the wavelet scalogram $\mathbf{I}(j)$.

Remark. In Fourier analysis, e.g. Shimotsu (2007), the periodogram is normalized. In wavelet analysis, the normalization factor may depend on the resolution $j$, and the scalogram is not normalized. For every $j$ the scalogram $\mathbf{I}(j)$ should be normalized by $n_{j}$. In the remainder of the paper, we will keep the initial $\mathbf{I}(j)$ for convenience.

Deriving expression (7) with respect to the matrix $\mathbf{G}$ yields

$$
\frac{\partial \mathcal{L}}{\partial \mathbf{G}}(\mathbf{G}, \mathbf{d})=\frac{1}{n} \sum_{j=j_{0}}^{j_{1}}\left[n_{j} \mathbf{G}^{-1}-\mathbf{G}^{-1} \boldsymbol{\Lambda}_{j}(\mathbf{d})^{-1} \mathbf{I}(j) \boldsymbol{\Lambda}_{j}(\mathbf{d})^{-1} \mathbf{G}^{-1}\right] .
$$

Hence, the minimum for fixed $\mathbf{d}$ is attained at

$$
\widehat{\mathbf{G}}(\mathbf{d})=\frac{1}{n} \sum_{j=j_{0}}^{j_{1}} \boldsymbol{\Lambda}_{j}(\mathbf{d})^{-1} \mathbf{I}(j) \boldsymbol{\Lambda}_{j}(\mathbf{d})^{-1}
$$


Replacing $\mathbf{G}(\mathbf{d})$ by $\widehat{\mathbf{G}}(\mathbf{d})$, the objective criterion is defined as

$$
\begin{aligned}
R(\mathbf{d}) & :=\mathcal{L}(\widehat{\mathbf{G}}(\mathbf{d}), \mathbf{d})-1 \\
& =\log \operatorname{det}(\widehat{\mathbf{G}}(\mathbf{d}))+\frac{1}{n} \sum_{j=j_{0}}^{j_{1}} n_{j} \log \left(\operatorname{det}\left(\boldsymbol{\Lambda}_{j}(\mathbf{d}) \boldsymbol{\Lambda}_{j}(\mathbf{d})\right),\right. \\
& =\log \operatorname{det}(\widehat{\mathbf{G}}(\mathbf{d}))+2 \log (2)\left(\frac{1}{n} \sum_{j=j_{0}}^{j_{1}} j n_{j}\right)\left(\sum_{\ell=1}^{p} d_{\ell}\right) .
\end{aligned}
$$

The vector of the long-memory parameters $\mathbf{d}$ is estimated by $\widehat{\mathbf{d}}=\operatorname{argmin} R(\mathbf{d})$. The estimator $\widehat{\mathbf{d}}$ is exactly equal to the one introduced in Moulines et al (2008) when the matrix $\Omega$ is diagonal corresponding to univariate setting.

In a second step of estimation we define $\widehat{\mathbf{G}}(\widehat{\mathbf{d}})$, estimator of $\mathbf{G}(\mathbf{d})$. Finally applying the correction of phase-shift yields the estimation of the covariance matrix $\Omega$

$$
\widehat{\Omega}_{\ell, m}=\widehat{G}_{\ell, m}(\widehat{\mathbf{d}}) /\left(\cos \left(\pi\left(\widehat{d}_{\ell}-\widehat{d}_{m}\right) / 2\right) K\left(\widehat{d}_{\ell}+\widehat{d}_{m}\right)\right) .
$$

Equation (10) is correctly defined as the probability that $\widehat{d}_{\ell}-\widehat{d}_{m}$ is exactly congruent to 1 modulo 2 is null. Consequently estimator $\widehat{\Omega}$ is defined almost surely. Yet, empirically when $d_{\ell}-d_{m}$ is close to 1 modulo 2 , the estimation of $\Omega$ may be strongly biased.

\section{Main results}

In the above, we have defined the MWW estimator, the following section deals with the asymptotic behaviour of the estimators. The consistency of the estimators is established, under a condition which controls the variance of the empirical wavelet cross-covariances. The first part of this section introduces this condition and characterizes a class of processes for which it is satisfied. The second part details the asymptotic results of convergence.

\subsection{Additional condition}

The following condition is an additional assumption, which gives an asymptotic control of the wavelet scalogram.

\section{Condition (C)}

$$
\text { For all } \ell, m=1, \ldots, p, \quad \sup _{n} \sup _{j \geqslant 0} \frac{1}{n_{j} 2^{2 j\left(d_{\ell}+d_{m}\right)}} \operatorname{Var}\left(I_{\ell, m}(j)\right)<\infty
$$

This condition is slightly more restrictive than condition (9) of Moulines et al (2008) in a univariate framework, where their spectral density of the process is only defined on a neighbourhood of zero. 
The following proposition gives a class of multivariate processes such that Condition (C) holds.

Proposition 4. Suppose that there exists a sequence $\{\mathbf{A}(u)\}_{u \in \mathbb{Z}}$ in $\mathbb{R}^{p \times p}$ such that $\sum_{u}\|\mathbf{A}(u)\|_{\infty}^{2}<\infty$ and

$$
\forall t, \quad \Delta^{D} \mathbf{X}(t)=\mu+\sum_{u \in \mathbb{Z}} \mathbf{A}(t+u) \varepsilon(t)
$$

with $\varepsilon(t)$ weak white noise process, in $\mathbb{R}^{p}$. Let $\mathcal{F}_{t-1}$ denote the $\sigma$-field of events generated by $\{\varepsilon(s), s \leqslant t-1\}$. Assume that $\varepsilon$ satisfies $\mathbb{E}\left[\varepsilon(t) \mid \mathcal{F}_{t-1}\right]=0, \mathbb{E}\left[\varepsilon_{a}(t) \varepsilon_{b}(t) \mid \mathcal{F}_{t-1}\right]=\mathbb{1}_{a=b}$ and $\mathbb{E}\left[\varepsilon_{a}(t) \varepsilon_{b}(t) \varepsilon_{c}(t) \varepsilon_{d}(t) \mid \mathcal{F}_{t-1}\right]=\mu_{a, b, c, d}$ with $\left|\mu_{a, b, c, d}\right| \leqslant \mu_{\infty}<\infty$, for all $a, b, c, d=1, \ldots, p$.

Then, under assumptions (W1)-(W5), Condition (C) holds.

The proof is given in appendix B.

This assumption of a Cramer-Wold type decomposition of the process $\mathbf{X}$ with a linear fourth-order stationary process was made among others by Lobato (1999), Shimotsu (2007), Giraitis et al (1997), or Theorem 1 of Moulines et al (2008). As discussed in Lobato (1999), there exist models with density (1), where the condition (C) is not satisfied, however, it is not particularly restrictive.

\subsection{Convergence}

We suppose that we have $N$ observations of a multivariate $p$-vector process $\mathbf{X}$, namely $\mathbf{X}(1), \ldots \mathbf{X}(N)$ with a spectral density satisfying approximation (2) around the zero frequency. For given functions $(\phi(\cdot), \psi(\cdot))$, and for given levels $0 \leqslant j_{0} \leqslant j_{1}$, the estimator of $\mathbf{d}$ is the argument minimizing $R$, defined by (9), and the matrix $\mathbf{G}$ is estimated by $\widehat{\mathbf{G}}(\widehat{\mathbf{d}})$ defined by (8). From now on, we will add the superscript 0 to denote the true parameter values, $\mathbf{d}^{0}$ and $\mathbf{G}^{0}$.

The following assumptions on the resolution levels $j_{0}$ and $j_{1}$ will be needed throughout the paper. We assume that either the difference $j_{1}-j_{0}$ is constant or it tends to infinity as $N$ tends to infinity.

The following result shows the consistency of the estimators and the rate of convergence. The proofs are given in Appendix.

Theorem 5. Assume that (W1)-(W5) and Condition (C) hold. If $j_{0}$ and $j_{1}$ are chosen such that $2^{-j_{0} \beta}+$ $N^{-1 / 2} 2^{j_{0} / 2} \rightarrow 0$ and $j_{0}<j_{1} \leqslant j_{N}$ with $j_{N}=\max \left\{j, n_{j} \geqslant 1\right\}$, then

$$
\widehat{\mathbf{d}}-\mathbf{d}^{0}=o_{\mathbb{P}}(1) .
$$

This result generalises that of Moulines et al (2008). It deals with multivariate settings, with the same assumption on the wavelet filter and on the choice of the scale $j_{0}$. The condition in Theorem 5 is equal to the one obtained in Proposition 9 of Moulines et al (2008), in the univariate case, that is $1 / j_{0}+N^{-1 / 2} 2^{j_{0} / 2} \rightarrow 0$. We choose here to express the condition with the parameter $\beta$ since the optimal choice of $j_{0}$ depends on $\beta$ as it is shown in Corollary 7 below.

The convergence of $\widehat{\mathbf{G}}(\widehat{d})$ to $\mathbf{G}^{0}$ is not established under assumptions of Theorem 5 . However, we prove it in the following theorem, under more restrictive conditions. 
Theorem 6. Assume that (W1)-(W5) and Condition (C) hold. If $j_{0}$ and $j_{1}$ are chosen such that $\log (N)^{2}\left(2^{-j_{0} \beta}+N^{-1 / 2} 2^{j_{0} / 2}\right) \rightarrow 0$ and $j_{0}<j_{1} \leqslant j_{N}$ then

$$
\begin{aligned}
\widehat{\mathbf{d}}-\mathbf{d}^{0}=O_{\mathbb{P}}\left(2^{-j_{0} \beta}+\right. & \left.N^{-1 / 2} 2^{j_{0} / 2}\right), \\
\forall(\ell, m) \in\{1, \ldots, p\}^{2}, \widehat{G}_{\ell, m}(\widehat{\mathbf{d}})-G_{\ell, m}\left(\mathbf{d}^{0}\right) & =O_{\mathbb{P}}\left(\log (N)\left(2^{-j_{0} \beta}+N^{-1 / 2} 2^{j_{0} / 2}\right)\right), \\
\widehat{\Omega}_{\ell, m}-\Omega_{\ell, m} & =O_{\mathbb{P}}\left(\log (N)\left(2^{-j_{0} \beta}+N^{-1 / 2} 2^{j_{0} / 2}\right)\right) .
\end{aligned}
$$

The condition in Theorem 6 is slightly different from Theorem 3 of Moulines et al (2008). Our result presents an additional $\log (N)$ term due to technical simplifications in the proof. However, it may be suppressed by adding technical details. The same arguments apply for the $\log (N)$ term appearing in the rate of the convergence of the matrix.

The optimal rate is then expressed by balancing the two terms appearing in the bound above.

Corollary 7. Assume that (W1)-(W5) and Condition (C) hold. Taking $2^{j_{0}}=N^{1 /(1+2 \beta)}$,

$$
\widehat{\mathbf{d}}-\mathbf{d}^{0}=O_{\mathbb{P}}\left(N^{-\beta /(1+2 \beta)}\right) .
$$

This corresponds to the optimal rate (Giraitis et al, 1997). Fourier Whittle estimators in Lobato (1999) and Shimotsu (2007) obtained the rate $m^{1 / 2}$ where $m$ is the number of discrete frequencies used in the Fourier transform. When $m \sim c N^{\zeta}$ with a positive constant $c$, the convergence is obtained for $0<\zeta<2 \beta /(1+2 \beta)$. Wavelet estimators thus give a slightly better rate of convergence.

Result of Corollary 7 stresses that it is necessary to fix the finest frequency $j_{0}$ in the wavelet procedure at a given scale depending on the regularity $\beta$ of the density $f^{\mathcal{S}}(\cdot)$. A possible extension is to develop an estimation which is adaptive relatively to the parameter $\beta$. This is done e.g. in univariate Fourier analysis by Iouditsky et al (2001). However, this topic exceeds the scope of this paper.

Further results on asymptotic normality, and in particular the asymptotic variance of the estimators, would give important information to quantify the quality of the estimators. In particular it would give a theoretical mean of comparison between the Fourier-based and the wavelet-based approaches or between the univariate and the multivariate estimations of $\mathbf{d}$. This work is in progress and will be established in a future paper. Here, the comparison is done with a simulation study.

\section{Simulations}

In this section, simulated data are used to study the behaviour of the proposed procedure using one illustrative example. An extensive simulation study would exceed the scope of this paper, and will be provided in a future paper. Here, we consider an $\operatorname{ARFIMA}(0, \mathbf{d}, 0)$ with a long-run correlation 
matrix $\Omega=\left(\begin{array}{ll}1 & \rho \\ \rho & 1\end{array}\right)$ and $\rho=0.4$. The proposed multivariate wavelet Whittle (MWW) estimators are computed for $N=512$ observations and 1000 Monte-Carlo replications. A R package named multiwave is available and Matlab codes are available on request.

A set of different values of $\mathbf{d}$ is considered. The choices are restricted to settings where the two components of the processes share the same order of stationarity. Indeed, it seems natural that time series measuring similar phenomena have similar stationary properties. We simulated both stationary and nonstationary ARFIMA processes, $d_{1}=0.2$ and $d_{1}=1.2$. Our MWW estimator is shown to be consistent and the quality increases when $|\rho|$ increases. We also conducted a comparison between our estimators and multivariate Fourier Whittle (MFW) estimators developed by Shimotsu (2007) for stationary processes only. In nonstationary cases, Nielsen (2011) proposed a similar approach based on the extended Fourier transform of Abadir et al (2007). However in our simulations, this approach gives satisfactory results only for $\mathbf{d}<1.5$.

A wavelet-based procedure with $M$ vanishing moments should be compared with an estimation based on tapered Fourier of order M. Such a comparison has been driven in Faÿ et al (2009) in one-dimensional settings. The authors established that wavelet-based estimation outperforms tapered Fourier estimation. Similar observations are expected in multivariate framework. Yet as multivariate Whittle estimation based on tapered Fourier transform has not been studied in literature, we choose not to display such a comparison.

It is worth pointing out that the main advantage of wavelets is their flexibility. Wavelet-based estimators can be applied for a large set of data, whatever the degree of stationarity is (if still smaller than the number of vanishing moments) and even if the processes contain polynomial trends which is very attractive for real data applications.

\section{Parameters used for estimation.}

The quality of estimation by wavelets relies on the choice of the wavelet bases. A trade-off is necessary between the number of vanishing moments and the support size of the wavelets. In time series analysis, the number of vanishing moments enables to consider polynomial trends or nonstationary time series, due to the constraint $\sup _{\ell} d_{\ell} \leqslant M$. Yet, the support size of the wavelet is proportional to the number of vanishing moments and increases the variance of estimation.

The wavelet basis used in this section is the Daubechies wavelet with $M=4$ vanishing moments. Its regularization parameter is $\alpha=1.91$. In our framework, when considering stationary time series, we could also apply our procedure using Haar bases. Estimation based on Haar wavelet indeed gives better results, possibly better than Fourier (see e.g. Gencay and Signori (2015) in the case of tests of serial correlation). As explained above a lower number of vanishing moments improves the quality of the wavelet-based estimators, see Faÿ et al (2009) in the univariate case. Similar results are observed in multivariate estimations. They are not presented here for the sake of concision. As our goal is to propose a flexible method for real data application, we prefer to consider a higher vanishing moments bases to stress its flexibility.

The method is controlled by the scales $j_{0}$ and $j_{1}$. The scale $j_{1}$ is fixed equal to $\log _{2}(N)$ while $j_{0}$ is chosen so that the optimal mean square error is minimal. Increasing $j_{0}$ leads to a smaller bias but a 
higher variance since less coefficients are used in the estimation process, which may be controlled by an adaptive procedure. As stated by Theorem 5, the finest scales have to be removed from estimation to get rid of the presence of the short-range dependence $f^{\mathcal{S}}(\cdot)$. Similar considerations can be found in Achard et al (2008) and Faÿ et al (2009).

Concerning MFW estimation, the main parameter is the number $m$ of frequencies used in the procedure. An usual choice in literature is $m=N^{0.65}$ (see e.g. Shimotsu (2007) or Nielsen and Frederiksen (2005)). Additionally MFW estimators are evaluated using values of $m$ giving the same number of Fourier coefficients than of wavelet coefficients. The final $m$ kept is the one giving the optimal mean square error. The parallel between the number of wavelet scales and the number of Fourier frequencies has been discussed in Faÿ et al (2009).

\section{Measures of quality.}

The quality of the estimators is measured by the bias, the standard deviation (std) and the RMSE which is equal to the square root of $\left(b i a s^{2}+s t d^{2}\right)$. In order to display an easy comparison between the univariate and the multivariate approaches, we compute the ratio between the RMSE obtained with the multivariate wavelet Whittle estimation and the RMSE obtained with univariate wavelet Whittle estimations. It is denoted by ratio $M / U$. A similar quantity is defined for the comparison with MFW estimation. We define ratio $W / S$ to be the ratio between the RMSE respectively using wavelet-based estimators and Fourier-based estimators.

\subsection{Estimation of the long-memory parameters $d$}

Results for the estimation of $\mathbf{d}$ are presented in Table 1 . The ratio $\mathrm{M} / \mathrm{U}$ points out that the quality of estimation is increased with the multivariate approach with respect to the univariate procedure. When the series are correlated, it is better to use MWW estimators to infer the long-memory parameters. The estimation is still satisfactory in nonstationary settings.

Table 2 displays the results of the MFW estimators described in Shimotsu (2007). With the usual number of frequencies $m=N^{0.65}$ in Fourier-based estimation, our wavelet-based procedure leads to lower RMSEs, as quantified by the ratio W/F. More precisely the good performance of our scheme of estimation is due to a lower variance, even if the bias is higher. With a higher number of frequencies in Fourier-based estimation, taking a value that minimizes the RMSE, the MWW estimators are no more preferable to MFW. Yet, the ratio W/F stays close to 1 and the analysis of the bias and variances reveals similar orders of magnitude.

\subsection{Estimation of the long-run covariance $\Omega$}

This section deals with the estimation of the long-run covariance matrix $\Omega$ and the estimation of the correlation $\Omega_{12} / \sqrt{\Omega_{11} \Omega_{22}}$. This latter quantity corresponds in literature to the power-law coherency between the two time series (Sela and Hurvich, 2012) or to the fractal connectivity (Achard et al, 2008). 
Table 1: Multivariate Whittle wavelet estimation of $\mathbf{d}$ for a bivariate $\operatorname{ARFIMA}(0, \mathbf{d}, 0)$ with $\rho=0.4$, $N=512$ with 1000 repetitions.

\begin{tabular}{rrcccc}
\hline \multicolumn{6}{c}{$j_{0}=1}$. \\
\hline$d_{1}$ & $\mathbf{d}$ & bias & std & RMSE & ratio M/U \\
\hline \hline 0.2 & 0.2 & -0.0267 & 0.0413 & 0.0492 & 0.9080 \\
& -0.2 & 0.0379 & 0.0430 & 0.0574 & 1.0595 \\
\hline 0.2 & -0.0298 & 0.0428 & 0.0522 & 0.9631 \\
0.0 & -0.0002 & 0.0438 & 0.0438 & 0.9504 \\
\hline 0.2 & -0.0330 & 0.0456 & 0.0563 & 0.9713 \\
0.2 & -0.0333 & 0.0443 & 0.0554 & 0.9831 \\
\hline 0.2 & -0.0304 & 0.0429 & 0.0526 & 0.9583 \\
0.4 & -0.0571 & 0.0461 & 0.0734 & 0.9701 \\
\hline
\end{tabular}

\begin{tabular}{cccccc}
\hline \multicolumn{6}{c}{$j_{0}=2}$. \\
\hline$d_{1}$ & $\mathbf{d}$ & bias & std & RMSE & ratio M/U \\
\hline \hline 1.2 & 1.2 & -0.0380 & 0.0830 & 0.0913 & 0.9728 \\
& 0.8 & -0.0298 & 0.0775 & 0.0831 & 0.9643 \\
\hline & 1.2 & -0.0360 & 0.0818 & 0.0894 & 0.9702 \\
1.0 & -0.0346 & 0.0808 & 0.0879 & 0.9626 \\
\hline & 1.2 & -0.0463 & 0.0853 & 0.0970 & 0.9677 \\
1.2 & -0.0393 & 0.0850 & 0.0936 & 0.9688 \\
\hline & 1.2 & -0.0369 & 0.0799 & 0.0880 & 0.9589 \\
1.4 & -0.0482 & 0.0863 & 0.0989 & 0.9648 \\
\hline
\end{tabular}

Table 2: Multivariate Whittle Fourier estimation of $\mathbf{d}$ for a bivariate $\operatorname{ARFIMA}(0, \mathbf{d}, 0)$ with $\rho=0.4$, $N=512$ with 1000 repetitions. Two number of frequencies $m$ are presented: the usual choice $m=\left\lfloor N^{0.65}\right\rfloor$ and the value giving the lower RMSE. $\lfloor x\rfloor$ denotes the closest integer smaller than $x$.

\begin{tabular}{rcccc}
\hline \multicolumn{5}{c}{$m=\left\lfloor N^{0.65}\right\rfloor=57}$. \\
\hline d & bias & std & RMSE & ratio W/F \\
\hline \hline 0.2 & -0.0087 & 0.0707 & 0.0712 & 0.6908 \\
-0.2 & -0.0001 & 0.0824 & 0.0824 & 0.6958 \\
\hline 0.2 & -0.0037 & 0.0679 & 0.0680 & 0.7674 \\
0.0 & -0.0010 & 0.0778 & 0.0778 & 0.5630 \\
\hline 0.2 & -0.0078 & 0.0691 & 0.0695 & 0.8101 \\
0.2 & -0.0043 & 0.0733 & 0.0735 & 0.7546 \\
\hline 0.2 & -0.0038 & 0.0705 & 0.0706 & 0.7445 \\
0.4 & 0.0012 & 0.0788 & 0.0788 & 0.9320 \\
\hline
\end{tabular}

\begin{tabular}{rcccc}
\hline \multicolumn{5}{c}{$m=\left\lfloor N^{0.876}\right\rfloor=236}$. \\
\hline $\mathbf{d}$ & bias & std & RMSE & ratio W/F \\
\hline \hline 0.2 & -0.0174 & 0.0318 & 0.0362 & 1.3581 \\
-0.2 & 0.0158 & 0.0323 & 0.0359 & 1.5964 \\
\hline 0.2 & -0.0170 & 0.0315 & 0.0358 & 1.4558 \\
0.0 & -0.0025 & 0.0318 & 0.0319 & 1.3728 \\
\hline 0.2 & -0.0200 & 0.0321 & 0.0378 & 1.4875 \\
0.2 & -0.0189 & 0.0320 & 0.0372 & 1.4905 \\
\hline 0.2 & -0.0201 & 0.0325 & 0.0382 & 1.3759 \\
0.4 & -0.0317 & 0.0366 & 0.0484 & 1.5169 \\
\hline
\end{tabular}


Table 3: Wavelet Whittle estimation of $\Omega$ for a bivariate $\operatorname{ARFIMA}(0, \mathbf{d}, 0)$ with $\rho=0.4, N=512$ with 1000 repetitions.

\begin{tabular}{lcccc}
\hline \multicolumn{5}{c}{$j_{0}=1}$. \\
\hline $\mathbf{d}$ & & bias & std & RMSE \\
\hline \hline$(0.2,-0.2)$ & $\Omega_{1,1}$ & 0.0342 & 0.0710 & 0.0788 \\
& $\Omega_{1,2}$ & 0.0387 & 0.0605 & 0.0718 \\
& $\Omega_{2,2}$ & -0.0402 & 0.0709 & 0.0815 \\
& correlation & 0.0400 & 0.0496 & 0.0637 \\
\hline$(0.2,0.0)$ & $\Omega_{1,1}$ & 0.0309 & 0.0697 & 0.0762 \\
& $\Omega_{1,2}$ & 0.0176 & 0.0540 & 0.0568 \\
& $\Omega_{2,2}$ & -0.0012 & 0.0732 & 0.0733 \\
& correlation & 0.0113 & 0.0417 & 0.0432 \\
\hline$(0.2,0.2)$ & $\Omega_{1,1}$ & 0.0297 & 0.0733 & 0.0790 \\
& $\Omega_{1,2}$ & 0.0116 & 0.0518 & 0.0530 \\
& $\Omega_{2,2}$ & 0.0282 & 0.0725 & 0.0778 \\
& correlation & -0.0003 & 0.0386 & 0.0386 \\
\hline $0.2,0.4)$ & $\Omega_{1,1}$ & 0.0356 & 0.0703 & 0.0788 \\
& $\Omega_{1,2}$ & 0.0328 & 0.0568 & 0.0655 \\
& $\Omega_{2,2}$ & 0.0707 & 0.0728 & 0.1015 \\
& correlation & 0.0106 & 0.0422 & 0.0435 \\
\hline
\end{tabular}

\begin{tabular}{lcccc}
\hline \multicolumn{5}{c}{$j_{0}=2}$. \\
\hline $\mathbf{d}$ & & bias & std & RMSE \\
\hline \hline$(1.2,0.8)$ & $\Omega_{1,1}$ & 0.0037 & 0.1473 & 0.1474 \\
& $\Omega_{1,2}$ & 0.0478 & 0.1199 & 0.1290 \\
& $\Omega_{2,2}$ & 0.0052 & 0.1303 & 0.1304 \\
& correlation & 0.0462 & 0.1041 & 0.1139 \\
\hline$(1.2,1.0)$ & $\Omega_{1,1}$ & -0.0031 & 0.1411 & 0.1411 \\
& $\Omega_{1,2}$ & 0.0182 & 0.1003 & 0.1019 \\
& $\Omega_{2,2}$ & 0.0027 & 0.1357 & 0.1357 \\
& correlation & 0.0176 & 0.0781 & 0.0800 \\
\hline$(1.2,1.2)$ & $\Omega_{1,1}$ & 0.0055 & 0.1442 & 0.1443 \\
& $\Omega_{1,2}$ & 0.0060 & 0.0921 & 0.0923 \\
& $\Omega_{2,2}$ & -0.0033 & 0.1456 & 0.1456 \\
& correlation & 0.0052 & 0.0685 & 0.0687 \\
\hline$(1.2,1.4)$ & $\Omega_{1,1}$ & 0.0001 & 0.1496 & 0.1496 \\
& $\Omega_{1,2}$ & 0.0155 & 0.1039 & 0.1051 \\
& $\Omega_{2,2}$ & 0.0135 & 0.1610 & 0.1615 \\
& correlation & 0.0125 & 0.0802 & 0.0812
\end{tabular}

The results obtained in simulations for MWW estimation of the covariance and correlation are given in Table 3. The quality is satisfactory in all settings, especially in the stationary ones.

The results for MFW estimation are displayed in Table 4. When MFW is applied with $m=N^{0.65}$ frequencies, the ratio $\mathrm{W} / \mathrm{F}$ is less than 1 . Like for the estimation of $\mathbf{d}$ the good performance of MWW estimators is principally due to a smaller variance. When MFW estimators are implemented with a higher number of frequencies, giving optimal results for the estimation of $\mathbf{d}$, the difference between MWW and MFW procedures decreases. The quality of the two estimation schemes are similar, with comparable values for bias and variances.

To conclude, the multivariate approach increases the quality of estimation of the long-memory parameters $\mathbf{d}$ in comparison with a univariate estimation. In stationary frameworks, the performance is very similar to multivariate Fourier Whittle estimation, when estimating the vector $\mathbf{d}$ or the long-run covariance matrix. The main advantage of our wavelet-based procedure is then its flexibility. By contrast with Fourier-based estimation, our estimators can be applied in a larger scope of situations, with nonstationary processes or in the presence of polynomial trends in the time series. 
Table 4: Fourier Whittle estimation of $\Omega$ for a bivariate $\operatorname{ARFIMA}(0, \mathbf{d}, 0)$ with $\rho=0.4, N=512$ with 1000 repetitions. Two number of frequencies $m$ are presented: the usual choice $m=\left\lfloor N^{0.65}\right\rfloor$ and the value giving the lower RMSE.

\begin{tabular}{lccccc}
\hline \multicolumn{5}{c}{$m=\left\lfloor N^{0.65}\right\rfloor=57}$. \\
\hline $\mathbf{d}$ & \multicolumn{1}{c}{ bias } & std & RMSE & ratio W/F \\
\hline \hline$(0.2,-0.2)$ & $\Omega_{1,1}$ & 0.0394 & 0.2253 & 0.2287 & 0.3444 \\
& $\Omega_{1,2}$ & 0.0091 & 0.1156 & 0.1160 & 0.6189 \\
& $\Omega_{2,2}$ & 0.0145 & 0.2308 & 0.2313 & 0.3525 \\
& correlation & -0.0002 & 0.0774 & 0.0774 & 0.8229 \\
\hline$(0.2,0.0)$ & $\Omega_{1,1}$ & 0.0245 & 0.2245 & 0.2259 & 0.3373 \\
& $\Omega_{1,2}$ & 0.0124 & 0.1154 & 0.1161 & 0.4892 \\
& $\Omega_{2,2}$ & 0.0163 & 0.2341 & 0.2347 & 0.3121 \\
& correlation & 0.0061 & 0.0793 & 0.0795 & 0.5428 \\
\hline$(0.2,0.2)$ & $\Omega_{1,1}$ & 0.0319 & 0.2319 & 0.2341 & 0.3376 \\
& $\Omega_{1,2}$ & 0.0141 & 0.1191 & 0.1199 & 0.4423 \\
& $\Omega_{2,2}$ & 0.0236 & 0.2331 & 0.2343 & 0.3321 \\
& correlation & 0.0041 & 0.0781 & 0.0782 & 0.4935 \\
\hline $0.2,0.4)$ & $\Omega_{1,1}$ & 0.0264 & 0.2255 & 0.2271 & 0.3470 \\
& $\Omega_{1,2}$ & 0.0107 & 0.1232 & 0.1237 & 0.5298 \\
& $\Omega_{2,2}$ & 0.0276 & 0.2462 & 0.2478 & 0.4096 \\
& correlation & 0.0001 & 0.0783 & 0.0783 & 0.5548 \\
\hline
\end{tabular}

\begin{tabular}{cccc}
\hline \multicolumn{4}{c}{$m=\left\lfloor N^{0.876}\right\rfloor=236}$. \\
\hline bias & std & RMSE & ratio W/F \\
\hline \hline 0.0492 & 0.0679 & 0.0839 & 0.9395 \\
0.0009 & 0.0498 & 0.0498 & 1.4414 \\
-0.0470 & 0.0640 & 0.0794 & 1.0273 \\
0.0006 & 0.0387 & 0.0387 & 1.6464 \\
\hline 0.0449 & 0.0666 & 0.0803 & 0.9486 \\
0.0105 & 0.0506 & 0.0517 & 1.0985 \\
-0.0008 & 0.0677 & 0.0677 & 1.0819 \\
0.0014 & 0.0383 & 0.0383 & 1.1259 \\
\hline 0.0450 & 0.0708 & 0.0839 & 0.9417 \\
0.0176 & 0.0520 & 0.0549 & 0.9666 \\
0.0438 & 0.0690 & 0.0818 & 0.9517 \\
-0.0006 & 0.0382 & 0.0382 & 1.0099 \\
\hline 0.0489 & 0.0682 & 0.0839 & 0.9392 \\
0.0313 & 0.0531 & 0.0616 & 1.0632 \\
0.1052 & 0.0705 & 0.1267 & 0.8012 \\
0.0002 & 0.0384 & 0.0384 & 1.1307 \\
\hline
\end{tabular}




\section{Application on neuroscience data}

We apply our approach to neuroscience data where the recorded data are typical example of multivariate long-range dependent time series. Researchers are interested in characterising the brain connectivity. Usually, the connectivity is evaluated using correlations at different frequencies between time series measuring the brain activity. We will show in this section that our method is perfectly adequate to deal with these real data.

The study concerns MEG data acquired from a healthy 43 year old woman studied during rest with eyes open at the National Institute of Mental Health Bethesda, MD using a 274-channel CTF MEG system VSM MedTech, Coquitlam, BC, Canada operating at $600 \mathrm{~Hz}$. The data were previously used in Achard et al (2008). We consider $N=2^{15}$ time points for each of the 274 time series.

Figure 1 displays the time series for arbitrary four channels. It is clear that they present nonlinear trends. Consequently, Fourier methods are not adequate to analyse such data, and methods based on wavelets are better to use.

Figure 1: MEG recordings for 4 arbitrary channels.

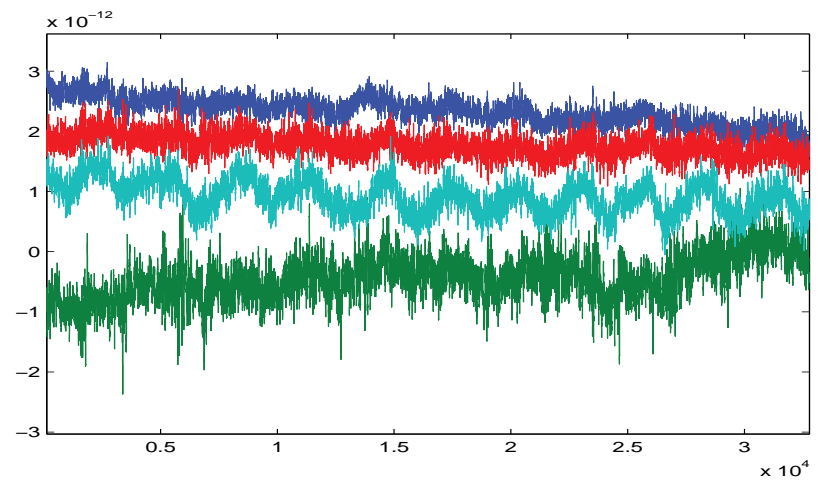

Our procedure was applied using scales 4 to 8 . It corresponds to frequencies between 1 to $20 \mathrm{~Hz}$. This choice was motivated by discussions with neuroscientists. It takes into account the presence of high-frequency noise which is modelled by $f^{\mathcal{S}}(\cdot)$. The data were preprocessed, and the lowfrequencies were removed. Figure 2 presents the results of the estimation of the long-memory parameters $\mathbf{d}$ and of the long-run covariance matrix $\Omega$.

First, the histogram of the estimate $\widehat{\mathbf{d}}$ shows that the maximal difference between the values of the long-memory parameters is less than 0.5 , and the problem of identifiability of $\Omega$ does not occur with these data. This allows us to give an estimate of the fractal connectivity. It is worth noticing that clusters appear in the correlation matrix. Most of them are situated along the diagonal, corresponding to spatially closed channels. Some channels are still correlated, even far from each others. It would be interesting to relate this result to a neuroscience interpretation. This will be investigated in future work. 
Figure 2: Results obtained by MWW estimators on the MEG dataset: histogram of the estimated long-memory parameters $\mathbf{d}$ (a) and estimated fractal connectivity matrix (b).

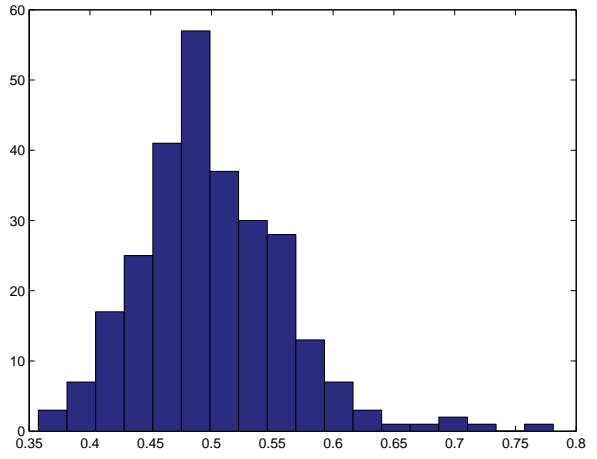

(a)

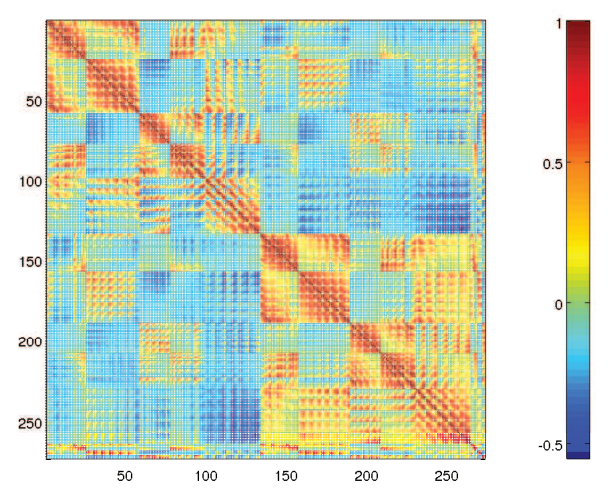

(b)

\section{Conclusion}

Many application fields are concerned with high-dimensional time series. A challenge is to characterize their long-memory properties and their correlation structure. The present work consider a semiparametric multivariate model, including a large class of multivariate processes such as some fractionally integrated processes. We propose an estimation of the long-dependence parameters and of the fractal connectivity, based on the Whittle approximation and on a wavelet representation of the time series. The theoretical properties of the estimation show the asymptotic optimality. A simulation study confirms that the estimation is well-behaved on finite samples. Finally we propose an application to the estimation of a human brain functional network based on MEG data sets. Our study highlights the benefit of the multivariate analysis, namely improved efficiency of estimation of dependence parameters and estimation of long-term correlations. Future work may concern the asymptotic normality of the estimators, since the development of tests may present a significant benefit for real data applications.

Acknowledgements. The authors thank the Co-Editor and two anonymous referees for their comments that led to substantial improvements in the paper. This work was partly supported by the IXXI research institute and the ANR project GRAPHSIP ANR-14-CE27-0001. This work has been done during the provisional assignment of I. Gannaz within CNRS at GIPSA-lab, Grenoble. The authors are grateful to Shimotsu (http://shimotsu.web.fc2.com/Site/Matlab_Codes.html) and to Faÿ, Moulines, Roueff and Taqqu for kindly providing the codes of their respective papers. 


\section{A Proof of Propositions 1, 2 and 3}

This section deals with the proof of Propositions 1 and 2. The proof of Proposition 3 is based on similar arguments and is omitted.

The covariance between $W_{j, k}(\ell)$ and $W_{j, k}(m)$ can be written with the cospectrum, $\theta_{\ell, m}(j)=$ $\int_{\mathbb{R}} \operatorname{Re}\left(f_{\ell, m}(\lambda)\right)\left|\mathbb{H}_{j}(\lambda)\right|^{2} d \lambda$. Indeed as the cross-spectral density is Hermitian, its imaginary part is an odd function,

$$
\theta_{\ell, m}(j)=\Omega_{\ell, m} \int_{\mathbb{R}}|2 \sin (\lambda / 2)|^{-\left(d_{\ell}+d_{m}\right)} \cos \left((\pi \operatorname{sign}(\lambda)-\lambda)\left(d_{\ell}-d_{m}\right) / 2\right) f_{\ell, m}^{S}(\lambda)\left|\mathbb{H}_{j}(\lambda)\right|^{2} d \lambda .
$$

The sinus function being odd,

$$
\theta_{\ell, m}(j)=\Omega_{\ell, m} \cos \left(\pi\left(d_{\ell}-d_{m}\right) / 2\right) \int_{\mathbb{R}}|2 \sin (\lambda / 2)|^{-\left(d_{\ell}+d_{m}\right)} \cos \left(\lambda\left(d_{\ell}-d_{m}\right) / 2\right) f_{\ell, m}^{S}(\lambda)\left|\mathbb{H}_{j}(\lambda)\right|^{2} d \lambda .
$$

The proof is very similar to Theorem 1 of Moulines et al (2007). Define the quantities $A_{\ell, m}(j)$ and $R_{\ell, m}(j)$,

$$
\begin{array}{lr}
A_{\ell, m}(j)=\Omega_{\ell, m} 2^{j} \cos \left(\pi\left(d_{\ell}-d_{m}\right) / 2\right) \int_{-\pi}^{\pi}|2 \sin (\lambda / 2)|^{-\left(d_{\ell}+d_{m}\right)} \cos \left(\lambda\left(d_{\ell}-d_{m}\right) / 2\right) \\
R_{\ell, m}(j)=\theta_{\ell, m}(j)-A_{\ell, m}(j) & f_{\ell, m}^{S}(\lambda)\left|\widehat{\phi}(\lambda) \widehat{\psi}\left(2^{j} \lambda\right)\right|^{2} d \lambda
\end{array}
$$

Following the proof of Moulines et al (2007), we can rewrite $A_{\ell, m}(j)$,

$$
\begin{aligned}
& A_{\ell, m}(j)=\Omega_{\ell, m} 2^{j} \cos \left(\pi\left(d_{\ell}-d_{m}\right) / 2\right) \\
& \qquad \int_{-\pi}^{\pi} g_{\ell, m}(\lambda)|\lambda|^{-\left(d_{\ell}+d_{m}\right)} \cos \left(\lambda\left(d_{\ell}-d_{m}\right) / 2\right) f_{\ell, m}^{S}(\lambda)|\widehat{\phi}(\lambda)|^{2}\left|\widehat{\psi}\left(2^{j} \lambda\right)\right|^{2} d \lambda \\
& \text { with } g_{\ell, m}(\lambda)=\left|\frac{2 \sin (\lambda / 2)}{\lambda}\right|^{-\left(d_{\ell}+d_{m}\right)} \text { for all } \lambda \in(-\pi, \pi) .
\end{aligned}
$$

- The assumption $f^{S}(\cdot) \in \mathcal{H}(\beta, L)$ states that $\left|f_{\ell, m}^{S}(\lambda)-1\right| \leqslant L|\lambda|^{\beta}$ for all $\lambda \in(-\pi, \pi)$.

- Under assumption (W1) the function $|\widehat{\phi}(\cdot)|^{2}$ is infinitely differentiable and bounded on $(-\pi, \pi)$.

- Using a Taylor expansion, the function $\mathbf{g}(\cdot)$ belongs to $\mathcal{H}\left(2, L_{g}\right)$ with $L_{g}=$ $\sup _{\ell, m=1, \ldots, p} \sup _{\lambda \in(-\pi, \pi)}\left|g_{\ell, m}{ }^{\prime \prime}(\lambda)\right|$ where $\mathbf{g}^{\prime \prime}(\cdot)$ denotes the second derivative of $\mathbf{g}(\cdot)$.

This implies that there exists a constant $C_{\phi, d}$ depending on $\phi(\cdot)$ and $\mathbf{d}$ such that

$$
\begin{array}{r}
\left.\left|A_{\ell, m}(j)-\Omega_{\ell, m} 2^{j} \cos \left(\pi\left(d_{\ell}-d_{m}\right) / 2\right) \int_{-\pi}^{\pi}\right| \lambda\right|^{-\left(d_{\ell}+d_{m}\right)} \cos \left(\lambda\left(d_{\ell}-d_{m}\right) / 2\right)\left|\widehat{\psi}\left(2^{j} \lambda\right)\right|^{2} d \lambda \mid \\
\leqslant C_{\phi, d} L 2^{j} \int_{-\pi}^{\pi}|\lambda|^{\left(\beta-d_{\ell}-d_{m}\right)}\left|\widehat{\psi}\left(2^{j} \lambda\right)\right|^{2} d \lambda
\end{array}
$$


With a change of variable,

$$
\begin{array}{r}
\left.\left|A_{\ell, m}(j)-\Omega_{\ell, m} 2^{j\left(d_{\ell}+d_{m}\right)} \cos \left(\pi\left(d_{\ell}-d_{m}\right) / 2\right) \int_{-2^{j} \pi}^{2^{j} \pi}\right| \lambda\right|^{-\left(d_{\ell}+d_{m}\right)} \cos \left(2^{-j} \lambda\left(d_{\ell}-d_{m}\right) / 2\right)|\widehat{\psi}(\lambda)|^{2} d \lambda \mid \\
\leqslant C_{\phi, d} L 2^{j\left(d_{\ell}+d_{m}-\beta\right)} \int_{-2^{j} \pi}^{-2^{j} \pi}|\lambda|^{\left(\beta-d_{\ell}-d_{m}\right)}|\widehat{\psi}(\lambda)|^{2} d \lambda .
\end{array}
$$

Under assumptions (W2), there exists a positive constant $C_{\psi}$ such that $\int_{-\pi}^{\pi}|\lambda|^{\left(\beta-d_{\ell}-d_{m}\right)}|\widehat{\psi}(\lambda)|^{2} d \lambda \leqslant$ $C_{\psi} \int_{-\infty}^{\infty}|\lambda|^{(\beta+2 \alpha)-d_{\ell}-d_{m}} d \lambda$. (W5) states that $(\beta+2 \alpha)-d_{\ell}-d_{m}<1$, the right-hand side of the inequality is bounded by a constant depending on $\psi(\cdot), \beta$ and $\min _{i=1, \ldots, p} d_{i}$. Using assumptions (W2) and (W5), we also have $\left.\left|\int_{|\lambda|>2^{j} \pi}\right| \lambda\right|^{-\left(d_{\ell}+d_{m}\right)} \cos \left(2^{-j} \lambda\left(d_{\ell}-d_{m}\right) / 2\right)|\widehat{\psi}(\lambda)|^{2} d \lambda \mid \leqslant$ $C_{\psi} \int_{|\lambda|>2^{j} \pi}|\lambda|^{-(1+\beta)} d \lambda$. The right-hand side is bounded by a constant depending on $\psi$ and $\beta$. We obtain that there exists a constant $C_{0}$ depending on $\alpha, \beta, \phi(\cdot), \psi(\cdot), \min _{i=1, \ldots, p} d_{i}$ and $\Omega_{\ell, m}$ such that

$$
\left|A_{\ell, m}(j)-\Omega_{\ell, m} 2^{j\left(d_{\ell}+d_{m}\right)} \cos \left(\pi\left(d_{\ell}-d_{m}\right) / 2\right) K_{j}\left(d_{\ell}+d_{m}\right)\right| \leqslant C_{0} L 2^{j\left(d_{\ell}+d_{m}-\beta\right)},
$$

with $K_{j}\left(d_{\ell}, d_{m}\right)=\int_{-\infty}^{\infty}|\lambda|^{-\left(d_{\ell}+d_{m}\right)} \cos \left(2^{-j} \lambda\left(d_{\ell}-d_{m}\right) / 2\right)|\widehat{\psi}(\lambda)|^{2} d \lambda$.

On the other hand, we can consider a first order approximation. Let

$$
\begin{gathered}
A_{\ell, m}(j)=\Omega_{\ell, m} 2^{j} \cos \left(\pi\left(d_{\ell}-d_{m}\right) / 2\right) \int_{-\pi}^{\pi} g_{\ell, m}(\lambda)|\lambda|^{-\left(d_{\ell}+d_{m}\right)} f_{\ell, m}^{S}(\lambda)|\widehat{\phi}(\lambda)|^{2}\left|\widehat{\psi}\left(2^{j} \lambda\right)\right|^{2} d \lambda \\
\text { with now } g_{\ell, m}(\lambda)=\left|\frac{2 \sin (\lambda / 2)}{\lambda}\right|^{-\left(d_{\ell}+d_{m}\right)} \cos \left(\lambda\left(d_{\ell}-d_{m}\right) / 2\right) \text { for all } \lambda \in(-\pi, \pi) .
\end{gathered}
$$

Then a similar approximation is obtained,

$$
\begin{aligned}
\mid A_{\ell, m}(j)-\Omega_{\ell, m} 2^{j\left(d_{\ell}+d_{m}\right)} \cos \left(\pi\left(d_{\ell}-d_{m}\right) / 2\right) \int_{-2^{j} \pi}^{2^{j} \pi} & |\lambda|^{-\left(d_{\ell}+d_{m}\right)}|\widehat{\psi}(\lambda)|^{2} d \lambda \mid \\
& \leqslant C_{\phi, d} L 2^{j\left(d_{\ell}+d_{m}-\beta\right)} \int_{-2^{j} \pi}^{2^{j} \pi}|\lambda|^{\left(\beta-d_{\ell}-d_{m}\right)}|\widehat{\psi}(\lambda)|^{2} d \lambda .
\end{aligned}
$$

Using assumptions (W2) and (W5),

$$
\left|A_{\ell, m}(j)-\Omega_{\ell, m} 2^{j\left(d_{\ell}+d_{m}\right)} \cos \left(\pi\left(d_{\ell}-d_{m}\right) / 2\right) K(\delta)\right| \leqslant C L 2^{j\left(d_{\ell}+d_{m}-\beta\right)},
$$

with $K(\delta)=\int_{-\infty}^{\infty}|\lambda|^{-(\delta)}|\widehat{\psi}(\lambda)|^{2} d \lambda$

Finally, $R_{\ell, m}(j)$ is bounded by $R_{\ell, m}(j) \leqslant C L 2^{\left(d_{\ell}+d_{m}-\beta\right) j}$. This inequality follows from the approximation of the squared gain function of the wavelet filter given in Proposition 3 of Moulines et al (2007) and from similar arguments to those given for $A_{\ell, m}(j)$. We do not detail the proof here for the sake of concision and we refer to the proof of Theorem 1 in Moulines et al (2007). 


\section{B Proof of Proposition 4}

Since the wavelet $\psi$ admits $M$ vanishing moments, at each scale $j \geqslant 0$, the associated filter $\mathbb{H}_{j}$ is factorised as $\mathbb{H}_{j}(\lambda)=\left(1-e^{i \lambda}\right)^{M} \widetilde{\mathbb{H}}_{j}(\lambda)$, with $\widetilde{\mathbb{H}}_{j}$ trigonometric polynomial, $\widetilde{\mathbb{H}}_{j}(\lambda)=\sum_{t \in \mathbb{Z}} \widetilde{h}_{j, t} e^{i t \lambda}$.

Since $M \geqslant D$, the wavelet coefficients may be written as

$$
W_{j, k}(\ell)=\sum_{t \in \mathbb{Z}} \widetilde{h}_{j, 2 j k-t}\left(\Delta^{D} X_{\ell}\right)(t)=\sum_{t \in \mathbb{Z}} \mathbf{B}_{\ell}\left(j, 2^{j} k-t\right) \varepsilon(t),
$$

where $\mathbf{B}_{\ell}\left(j, 2^{j} k-t\right)=\widetilde{h}_{j, 2^{j} k-t}\left(\Delta^{M-D} \mathbf{A}_{\ell}\right)(t)$. For all $\ell=1, \ldots, p$, the sequence $\left\{\mathbf{B}_{\ell}(j, u)\right\}_{u \in \mathbb{Z}}$ belongs to $\ell^{2}(\mathbb{Z})$.

We first give a preliminary result on the second order moment of $W_{j, k}(\ell)$,

$$
\mathbb{E}\left[W_{j, k}(\ell)^{2}\right]=\sum_{t, t^{\prime} \in \mathbb{Z} a, b=1, \ldots p} B_{\ell, a}\left(j, 2^{j} k-t\right) B_{\ell, b}\left(j, 2^{j} k-t^{\prime}\right) \mathbb{E}\left[\varepsilon_{a}(t) \varepsilon_{b}\left(t^{\prime}\right)\right] .
$$

Using the second-order properties of the process $\varepsilon$, the variance is equal to

$$
\mathbb{E}\left[W_{j, k}(\ell)^{2}\right]=\sum_{t \in \mathbb{Z}} \sum_{a=1, \ldots p} B_{\ell, a}\left(j, 2^{j} k-t\right)^{2} .
$$

Consider now $\mathbb{E}\left[I_{\ell, m}(j)^{2}\right]$,

$$
\begin{aligned}
& \mathbb{E}\left[I_{\ell, m}(j)^{2}\right]=\mathbb{E}\left[\left(\sum_{k} W_{j, k}(\ell) W_{j, k}(m)\right)^{2}\right] \\
&=\sum_{k, k^{\prime}, t^{\prime}, t^{\prime \prime}, t^{\prime \prime \prime} \in \mathbb{Z} a, b, c, d=1, \ldots p} B_{\ell, a}\left(j, 2^{j} k-t\right) B_{m, b}\left(j, 2^{j} k-t^{\prime}\right) B_{\ell, c}\left(j, 2^{j} k^{\prime}-t^{\prime \prime}\right) B_{m, d}\left(j, 2^{j} k^{\prime}-t^{\prime \prime \prime}\right) \\
& \mathbb{E}\left[\varepsilon_{a}(t) \varepsilon_{b}\left(t^{\prime}\right) \varepsilon_{c}\left(t^{\prime \prime}\right) \varepsilon_{d}\left(t^{\prime \prime \prime}\right)\right] .
\end{aligned}
$$

The fourth order behaviour of $\varepsilon$ implies that

$$
\begin{aligned}
\mathbb{E}\left[I_{\ell, m}(j)^{2}\right]= & \sum_{k, k^{\prime}} \sum_{t \in \mathbb{Z}} \sum_{a, b, c, d=1, \ldots . p} \mu_{a, b, c, d} B_{\ell, a}\left(j, 2^{j} k-t\right) B_{m, b}\left(j, 2^{j} k+t\right) B_{\ell, c}\left(j, 2^{j} k^{\prime}-t\right) B_{m, d}\left(j, 2^{j} k^{\prime}-t\right) \\
& +\sum_{k, k^{\prime}} \sum_{t \neq t^{\prime}} \sum_{a, b=1, \ldots p} B_{\ell, a}\left(j, 2^{j} k-t\right) B_{m, a}\left(j, 2^{j} k-t\right) B_{\ell, b}\left(j, 2^{j} k^{\prime}-t^{\prime}\right) B_{m, b}\left(j, 2^{j} k^{\prime}-t^{\prime}\right) \\
& +\sum_{k, k^{\prime}} \sum_{t \neq t^{\prime}} \sum_{a, b=1, \ldots p} B_{\ell, a}\left(j, 2^{j} k-t\right) B_{m, b}\left(j, 2^{j} k-t^{\prime}\right) B_{\ell, a}\left(j, 2^{j} k^{\prime}-t\right) B_{m, b}\left(j, 2^{j} k^{\prime}-t^{\prime}\right) \\
& +\sum_{k, k^{\prime}} \sum_{t \neq t^{\prime}} \sum_{a, b=1, \ldots p} B_{\ell, a}\left(j, 2^{j} k-t\right) B_{m, b}\left(j, 2^{j} k-t^{\prime}\right) B_{\ell, b}\left(j, 2^{j} k^{\prime}-t^{\prime}\right) B_{m, a}\left(j, 2^{j} k^{\prime}-t\right) .
\end{aligned}
$$

As $\mathbb{E}\left[I_{\ell, m}(j)\right]^{2}=\sum_{k, k^{\prime}} \sum_{t, t^{\prime}} \sum_{a, b} B_{\ell, a}\left(j, 2^{j} k-t\right) B_{m, a}\left(j, 2^{j} k-t\right) B_{\ell, b}\left(j, 2^{j} k^{\prime}-t^{\prime}\right) B_{m, b}\left(j, 2^{j} k^{\prime}-t^{\prime}\right)$, the 
variance of the scalogramm satisfies,

$$
\begin{aligned}
& \operatorname{Var}\left(I_{\ell, m}(j)\right) \\
= & \sum_{k, k^{\prime}} \sum_{t \in \mathbb{Z}} \sum_{a, b, c, d=1, \ldots p} \mu_{a, b, c, d} B_{\ell, a}\left(j, 2^{j} k-t\right) B_{m, b}\left(j, 2^{j} k-t\right) B_{\ell, c}\left(j, 2^{j} k^{\prime}-t\right) B_{m, d}\left(j, 2^{j} k^{\prime}-t\right) \\
& +\sum_{k, k^{\prime}} \mathbb{E}\left[W_{j, k}(\ell) W_{j, k^{\prime}}(\ell)\right] \mathbb{E}\left[W_{j, k}(m) W_{j, k^{\prime}}(m)\right]+\sum_{k, k^{\prime}} \mathbb{E}\left[W_{j, k}(\ell) W_{j, k^{\prime}}(m)\right] \mathbb{E}\left[W_{j, k}(m) W_{j, k^{\prime}}(\ell)\right] \\
& -\sum_{k, k^{\prime}} \sum_{t} \sum_{a, b=1, \ldots p} B_{\ell, a}\left(j, 2^{j} k-t\right) B_{m, a}\left(j, 2^{j} k-t\right) B_{\ell, b}\left(j, 2^{j} k^{\prime}-t\right) B_{m, b}\left(j, 2^{j} k^{\prime}-t\right) \\
& -\sum_{k, k^{\prime}} \sum_{t} \sum_{a, b=1, \ldots p} B_{\ell, a}\left(j, 2^{j} k-t\right) B_{m, b}\left(j, 2^{j} k-t\right) B_{\ell, a}\left(j, 2^{j} k^{\prime}-t\right) B_{m, b}\left(j, 2^{j} k^{\prime}-t\right) \\
& -\sum_{k, k^{\prime}} \sum_{t} \sum_{a, b=1, \ldots p} B_{\ell, a}\left(j, 2^{j} k-t\right) B_{m, b}\left(j, 2^{j} k-t\right) B_{\ell, b}\left(j, 2^{j} k^{\prime}-t\right) B_{m, a}\left(j, 2^{j} k^{\prime}-t\right) .
\end{aligned}
$$

Finally, $\operatorname{Var}\left(I_{\ell, m}(j)\right) \leqslant V_{1}+V_{2}+V_{3}$ with

$$
\begin{aligned}
& V_{1}=\left|\sum_{k, k^{\prime}} \mathbb{E}\left[W_{j, k}(\ell) W_{j, k^{\prime}}(\ell)\right] \mathbb{E}\left[W_{j, k}(m) W_{j, k^{\prime}}(m)\right]\right|, \\
& V_{2}=\left|\sum_{k, k^{\prime}} \mathbb{E}\left[W_{j, k}(\ell) W_{j, k^{\prime}}(m)\right] \mathbb{E}\left[W_{j, k}(m) W_{j, k^{\prime}}(\ell)\right]\right|, \\
& V_{3}=\left(1+\mu_{\infty}\right) \sum_{k, k^{\prime}} \sum_{t \in \mathbb{Z}} \sum_{a, b, c, d=1, \ldots p}\left|B_{\ell, a}\left(j, 2^{j} k-t\right) B_{m, b}\left(j, 2^{j} k-t\right) B_{\ell, c}\left(j, 2^{j} k^{\prime}-t\right) B_{m, d}\left(j, 2^{j} k^{\prime}-t\right)\right| .
\end{aligned}
$$

\section{Bounds $V_{1}$ and $V_{2}$}

Proposition 3 states that $\operatorname{Cov}\left(W_{j, k}(\ell), W_{j, k^{\prime}}(\ell)\right)=\int_{-\pi}^{\pi} e^{i \lambda\left(k-k^{\prime}\right)} D_{0 ; 0}^{(j)}(\lambda ;(\ell, \ell)) d \lambda$. The quantity $\int_{-\pi}^{\pi} e^{i \lambda v} D_{0 ; 0}^{(j)}(\lambda ;(\ell, \ell)) d \lambda$ is the $v$-th Fourier coefficient of the function $D_{0,0}^{(j)}\left(\cdot ; 2 d_{\ell}\right)$. Consequently, Parseval theorem implies that $\sum_{v \in \mathbb{Z}} \mathbb{E}\left[W_{j, k}(\ell) W_{j, k+v}(\ell)\right] \mathbb{E}\left[W_{j, k}(m) W_{j, k+v}(m)\right]$ converges to $I_{0}^{(j)}\left(2 d_{\ell}, 2 d_{m}\right)=\int_{-\pi}^{\pi} D_{0,0}^{(j)}(\lambda ;(\ell, \ell)) D_{0,0}^{(j)}(\lambda ;(m, m)) d \lambda$. The approximation given in Proposition 3 yields $D_{0,0}^{(j)}(\lambda ;(\ell, \ell)) \leqslant \Omega_{\ell, \ell} 2^{22 d_{\ell}} \widetilde{D}_{0,0}\left(\lambda ; 2 d_{\ell}\right)+C L \pi 2^{\left(2 d_{\ell}-\beta\right) j}$. Then, using Minkowski inequality

$$
\frac{1}{n_{j} 2^{2 j\left(d_{\ell}+d_{m}\right)}} V_{2} \leqslant 2\left(\Omega_{\ell, \ell}^{2} \widetilde{I}_{0}\left(2 d_{\ell}\right)+C^{2} L^{2} \pi^{2} 2^{-2 \beta j}\right)^{1 / 2}\left(\Omega_{m, m}^{2} \widetilde{I}_{0}\left(2 d_{m}\right)+C^{2} L^{2} \pi^{2} 2^{-2 \beta j}\right)^{1 / 2} .
$$

where $\widetilde{I}_{0}(\delta)=\int_{-\pi}^{\pi} \widetilde{D}_{0,0}(\lambda ; \delta)^{2} d \lambda$. It follows that $\frac{1}{n_{j} 2^{2 j\left(d_{\ell}+d_{m}\right)}} V_{1}$ is bounded by a constant independent of $j$ and depending only on $\mathbf{d}, \boldsymbol{\Omega}, \beta, \phi(\cdot)$ and $\psi(\cdot)$.

Similar arguments apply to $V_{2}$. Therefore $\frac{1}{n_{j} 2^{2 j\left(d_{\ell}+d_{m}\right)}} V_{2}$ is bounded by $\frac{\int_{-\pi}^{\pi} \widetilde{D}_{0,0}\left(\lambda ; d_{\ell}+d_{m}\right)^{2} d \lambda}{2^{2 j\left(d_{\ell}+d_{m}\right)}}$. By Proposition $3, \frac{1}{n_{2} 2^{2 j\left(d_{\ell}+d_{m}\right)}} V_{2}$ is bounded by a constant depending only on $\mathbf{d}, \Omega, \beta, \phi(\cdot)$ and $\psi(\cdot)$. 


\section{Bound $V_{3}$}

The quantity $V_{3}$ is equal to

$$
\left(1+\mu_{\infty}\right) \sum_{k} \sum_{\substack{t \in \mathbb{Z} \\ t^{\prime} \in\left\{t+2^{j}\left(k-k^{\prime}\right), k^{\prime} \in \mathbb{Z}\right\}}} \sum_{a, b, c, d=1, \ldots p}\left|B_{\ell, a}\left(j, 2^{j} k-t\right) B_{m, b}\left(j, 2^{j} k-t\right) B_{\ell, c}\left(j, 2^{j} k-t^{\prime}\right) B_{m, d}\left(j, 2^{j} k-t^{\prime}\right)\right| .
$$

Applying Minkowski inequality on $V_{3}$,

$$
\begin{aligned}
V_{3} \leqslant\left(1+\mu_{\infty}\right) & \sum_{k} \sum_{t \in \mathbb{Z}} \sum_{a, b=1, \ldots p}\left|B_{\ell, a}\left(j, 2^{j} k-t\right) B_{m, b}\left(j, 2^{j} k-t\right)\right| \\
& \left(\sum_{t^{\prime} \in\left\{t+2^{j} k^{\prime}, k^{\prime} \in \mathbb{Z}\right\}} \sum_{c, d=1, \ldots p} B_{\ell, c}\left(j, 2^{j} k-t^{\prime}\right)^{2}\right)^{1 / 2}\left(\sum_{t^{\prime} \in\left\{t-2^{j} k^{\prime}, k^{\prime} \in \mathbb{Z}\right\}} \sum_{c, d=1, \ldots p} B_{m, d}\left(j, 2^{j} k-t^{\prime}\right)^{2}\right)^{1 / 2} .
\end{aligned}
$$

Hence,

$$
\begin{aligned}
& V_{3} \leqslant\left(1+\mu_{\infty}\right) \sum_{k}\left(\sum_{t \in \mathbb{Z}} \sum_{a, b=1, \ldots p} B_{\ell, a}\left(j, 2^{j} k-t\right)^{2}\right)^{1 / 2}\left(\sum_{t \in \mathbb{Z}} \sum_{a, b=1, \ldots p} B_{m, b}\left(j, 2^{j} k-t\right)^{2}\right)^{1 / 2} \\
& \left(\sum_{t^{\prime} \in \mathbb{Z}} \sum_{c, d=1, \ldots p} B_{\ell, c}\left(j, 2^{j} k-t^{\prime}\right)^{2}\right)^{1 / 2}\left(\sum_{t^{\prime} \in \mathbb{Z}} \sum_{c, d=1, \ldots p} B_{m, d}\left(j, 2^{j} k-t^{\prime}\right)^{2}\right)^{1 / 2} .
\end{aligned}
$$

Together with (11) the following inequality is obtained, $V_{3} \leqslant\left(1+\mu_{\infty}\right) n_{j} p^{2} \theta_{\ell, \ell}(j) \theta_{m, m}(j)$. To conclude

$$
\frac{1}{n_{j} 2^{2 j\left(d_{\ell}+d_{m}\right)}} V_{1} \leqslant\left(1+\mu_{\infty}\right) p^{2} \frac{\theta_{\ell, \ell}(j) \theta_{m, m}(j)}{2^{2 j\left(d_{\ell}+d_{m}\right)}} .
$$

Condition (C) is proved since $\frac{\theta_{\ell, \ell}(j) \theta_{m, m}(j)}{2^{2 j\left(d_{\ell}+d_{m}\right)}}$ tends to a constant independent of $j$ thanks to Proposition 2.

\section{Preliminary results}

Let us take $\ell$ and $m$ in $1, \ldots, p$, and define, for any sequence $\mu=\left\{\mu_{j}, j \geqslant 0\right\}$,

$$
S_{\ell, m}(\mu)=\sum_{j, k} \mu_{j}\left(\frac{W_{j, k}(\ell) W_{j, k}(m)}{2^{j\left(d_{\ell}^{0}+d_{m}^{0}\right)}}-G_{\ell, m}^{0}\right)=\sum_{j=j_{0}}^{j_{1}} \mu_{j}\left(\frac{I_{\ell, m}(j)}{2^{j\left(d_{\ell}^{0}+d_{m}^{0}\right)}}-n_{j} G_{\ell, m}^{0}\right) .
$$

Proposition 8. Assume that the sequences $\mu$ belong to the set $\left\{\left\{\mu_{j}\right\}_{j} \geqslant 0,\left|\mu_{j}\right| \leqslant \frac{1}{n_{j}}\right\}$. Under condition (C), $\sup _{\left\{\mu,\left|\mu_{j}\right| \leqslant \frac{1}{n_{j}}\right\}} S_{\ell, m}(\mu)$ is uniformly bounded by $2^{-j_{0} \beta}+N^{-1 / 2} 2^{j_{1} / 2}$ up to a multiplicative constant, that is,

$$
\sup _{\mu \in\left\{\left(\mu_{j}\right)_{j \geqslant 0},\left|\mu_{j}\right| \leqslant \frac{1}{n_{j}}\right\}}\left\{S_{\ell, m}(\mu)\right\}=O_{\mathbb{P}}\left(2^{-j_{0} \beta}+N^{-1 / 2} 2^{j_{1} / 2}\right) .
$$


Proof. $S_{\ell, m}(\mu)$ is decomposed in two terms $S_{\ell, m}^{(0)}(\mu)$ and $S_{\ell, m}^{(1)}(\mu)$,

$$
\begin{aligned}
& S_{\ell, m}^{(0)}(\mu)=\sum_{j=j_{0}}^{j_{1}} \mu_{j} \frac{1}{2^{j\left(d_{\ell}^{0}+d_{m}^{0}\right)}} \sum_{k}\left(W_{j, k}(\ell) W_{j, k}(m)-\theta_{\ell, m}(j)\right), \\
& S_{\ell, m}^{(1)}(\mu)=\sum_{j=j_{0}}^{j_{1}} n_{j} \mu_{j}\left[\frac{\theta_{\ell, m}(j)}{2^{j\left(d_{\ell}^{0}+d_{m}^{0}\right)}}-G_{\ell, m}^{0}\right] .
\end{aligned}
$$

From Proposition 2,

$$
\begin{aligned}
& \left|S_{\ell, m}^{(0)}(\mu)\right| \leqslant \sum_{j=j_{0}}^{j_{1}}\left|\mu_{j}\right|\left|\sum_{k} W_{j, k}(\ell) W_{j, k}(m)-n_{j} \theta_{\ell, m}(j)\right|, \\
& \left|S_{\ell, m}^{(1)}(\mu)\right| \leqslant C \sum_{j=j_{0}}^{j_{1}} 2^{-\beta j} n_{j}\left|\mu_{j}\right| .
\end{aligned}
$$

Under the assumption $\left|\mu_{j}\right| \leqslant \frac{1}{n_{j}}$, we have the inequality $\left|S_{\ell, m}^{(1)}(\mu)\right| \leqslant C \sum_{j=j_{0}}^{j_{1}} 2^{-\beta j}$. The right-hand bound is equivalent to $2^{-j_{0} \beta}$ up to a constant. Condition (C) gives

$$
\mathbb{E}\left[\sup _{\left\{\mu,\left|\mu_{j}\right| \leqslant \frac{1}{n_{j}}\right\}}\left|S_{\ell, m}^{(0)}(\mu)\right|\right] \leqslant C^{\prime} \sum_{j=j_{0}}^{j_{1}} n_{j}^{-1 / 2},
$$

with a positive constant $C^{\prime}$. As $n_{j}=N 2^{-j}(1+o(1))$ the right-hand side of the inequality is equivalent to $C^{\prime} N^{-1 / 2} 2^{j_{1} / 2}$.

Proposition 9. Let $0<j_{0} \leqslant j_{1} \leqslant j_{N}$. Assume that the sequences $\mu$ belong to the set

$$
\mathcal{S}(q, \gamma, c)=\left\{\left\{\mu_{j}\right\}_{j \geqslant 0},\left|\mu_{j}\right| \leqslant \frac{c}{n}\left|j-j_{0}+1\right|^{q} 2^{\left(j-j_{0}\right)} \gamma \forall j=j_{0}, \ldots j_{1}\right\}
$$

with $0 \leqslant \gamma<1$. Under condition $(C), \sup _{\mu \in \mathcal{S}(q, \gamma, c)} S_{\ell, m}(\mu)$ is uniformly bounded by $2^{-j_{0} \beta}+$ $H\left(N^{-1 / 2} 2^{j_{0} / 2}\right)$ up to a constant,

$$
\sup _{\mu \in \mathcal{S}(q, \gamma, c)}\left\{S_{\ell, m}(\mu)\right\}=O_{\mathbb{P}}\left(2^{-j_{0} \beta}+H_{\gamma}\left(N^{-1 / 2} 2^{j_{0} / 2}\right)\right)
$$

with $H_{\gamma}(u)= \begin{cases}u & \text { if } 0 \leqslant \gamma<1 / 2 \\ \log \left(1+u^{-2}\right)^{q+1} u & \text { if } \gamma=1 / 2 \\ \log \left(1+u^{-2}\right)^{q} u^{2(1-\gamma)} & \text { if } 1 / 2<\gamma<1\end{cases}$

In particular, for any $0 \leqslant \gamma<1$, under the assumption $2^{-j_{0} \beta}+N^{-1 / 2} 2^{j_{0} / 2} \rightarrow 0$, we have $\sup _{\mu \in \mathcal{S}(q, \gamma, c)}\left\{S_{\ell, m}(\mu)\right\}=o_{\mathbb{P}}(1)$

Proof. Under the assumptions of the proposition, one deduce from inequality (14) that,

$$
\sup _{\mu \in \mathcal{S}(q, \gamma, c)}\left|S_{\ell, m}^{(1)}(\mu)\right| \leqslant c C \frac{1}{n} \sum_{j=j_{0}}^{j_{1}} n_{j} 2^{\left(-\beta j+\gamma\left(j-j_{0}\right)\right)}\left(j-j_{0}+1\right)^{q} \leqslant c C 2^{-\beta j_{0}} \sum_{i=0}^{j_{1}-j_{0}} 2^{-(1+\beta-\gamma) i}(i+1)^{q} .
$$


The sum on the right-hand side of the inequality tends to 0 because $1+\beta-\gamma>0$.

Similarly, under the additional Condition (C), inequality (13) is rewritten as,

$$
\begin{aligned}
\left.\mathbb{E}\left[\sup _{\mu \in \mathcal{S}(q, \gamma, c)} \mid S_{\ell, m}^{(0)}(\mu)\right) \mid\right] & \leqslant c C^{\prime} \frac{1}{n} \sum_{j=j_{0}}^{j_{1}} n_{j}^{1 / 2} 2^{\gamma\left(j-j_{0}\right)}\left(j-j_{0}+1\right)^{q} \\
& \leqslant c C^{\prime} N^{-1 / 2} 2^{j_{0} / 2} \sum_{i=0}^{j_{1}-j_{0}} 2^{-(1 / 2-\gamma) i}(i+1)^{q} .
\end{aligned}
$$

We distinguish three cases depending on the values of $\gamma$.

- The result is straightforward when $0 \leqslant \gamma<1 / 2$.

- If $\gamma=1 / 2$, the right-hand side is bounded by $c C^{\prime} N^{-1 / 2} 2^{j_{0} / 2}\left(j_{1}-j_{0}+1\right)^{q+1}$. The parameter $j_{1}$ satisfies $2^{j_{1}} \leqslant N$. Consequently $j_{1}-j_{0} \leqslant \log _{2}(N)+\log _{2}\left(2^{-j_{0}}\right)=\log _{2}\left(N 2^{-j_{0}}\right)$ and the result is proved.

- When $1 / 2<\gamma<1$, the right-hand side admits the upper bound $c C^{\prime \prime} N^{-1 / 2} 2^{j_{0} / 2}\left(j_{1}-j_{0}+\right.$ $1)^{q} 2^{(\gamma-1 / 2)\left(j_{1}-j_{0}\right)}$ with $C^{\prime \prime}$ a positive constant. Since $2^{j_{1}} \leqslant N$, it is inferior to $c C^{\prime \prime}\left(j_{1}-j_{0}+\right.$ $1)^{q}\left(N^{-1} 2^{j_{0}}\right)^{(1-\gamma)}$ which concludes the proof.

\section{Weak consistency}

We first establish the convergence under the condition $2^{-j_{0} \beta}+N^{-1 / 2} 2^{j_{1} / 2} \rightarrow 0$. This assumption is more restrictive than the condition $2^{-j_{0} \beta}+N^{-1 / 2} 2^{j_{0} / 2} \rightarrow 0$ given in Theorem 5 . Both conditions are equivalent when $j_{1}-j_{0}$ is finite but not in a general case.

We then prove Theorem 5 in two steps: first we establish a lower bound for $\widehat{\mathbf{d}}$ and second we develop a proof similar to the first one that has been given in section D.1 but with a weaker assumption thanks to the previous bound.

\section{D.1 Consistency under non-optimal assumptions}

In this section, we give a first result of consistency, with an assumption on $j_{0}$ and $j_{1}$ that can be weakened. This result is not necessary to obtain Theorem 5 but the scheme of the proof is similar and it points out why an additional step is necessary.

Proposition 10. Assume that (W1)-(W5) and Condition (C) hold. If in addition $j_{0}$ and $j_{1}$ are chosen such that $2^{-j_{0} \beta}+N^{-1 / 2} 2^{j_{1} / 2} \rightarrow 0$, then

$$
\begin{gathered}
\widehat{\mathbf{d}}-\mathbf{d}^{0}=o_{\mathbb{P}}(1), \\
\widehat{\mathbf{G}}(\widehat{\mathbf{d}})-\mathbf{G}\left(\mathbf{d}^{0}\right)=o_{\mathbb{P}}(1) .
\end{gathered}
$$


Proof. In order to evaluate the performances of the estimator of the long memory parameters, the first step consists in proving that the proposed estimator for $\mathbf{d}$ is consistent. The equivalent properties for $\Omega$ will be detailed in a second step. The proof is based on the following inequality,

$$
R(\mathbf{d})-R\left(\mathbf{d}^{0}\right) \geqslant L\left(\mathbf{d}-\mathbf{d}^{0}\right)+\Delta\left(\mathbf{d}, \mathbf{d}^{0}\right),
$$

where $L$ is a deterministic and convex function of $\mathbf{d}$ and the remaining term $\Delta$ tends uniformly to zero in probability.

We first establish inequality (15). The difference between the criterion evaluated at $\mathbf{d}$ and at the true long-memory parameters is equal to

$$
R(\mathbf{d})-R\left(\mathbf{d}^{0}\right)=\log \operatorname{det}(\widehat{\mathbf{G}}(\mathbf{d}))-\log \operatorname{det}\left(\widehat{\mathbf{G}}\left(\mathbf{d}^{0}\right)\right)+2 \log (2)<\mathcal{J}>\left(\sum_{\ell} d_{\ell}-d_{\ell}^{0}\right)
$$

where $<\mathcal{J}>=\frac{1}{n} \sum_{j=j_{0}}^{j_{1}} j n_{j}$ and $n=\sum_{j=j_{0}}^{j_{1}} n_{j}$.

The equality can be rewritten as

$$
\begin{aligned}
& R(\mathbf{d})-R\left(\mathbf{d}^{0}\right) \\
= & \log \operatorname{det}\left(\frac{1}{n} \sum_{j=j_{0}}^{j_{1}} \boldsymbol{\Lambda}_{<\mathcal{J}>}\left(\mathbf{d}-\mathbf{d}^{0}\right) \boldsymbol{\Lambda}_{j}(\mathbf{d})^{-1} I(j) \boldsymbol{\Lambda}_{j}(\mathbf{d})^{-1} \boldsymbol{\Lambda}_{<\mathcal{J}>}\left(\mathbf{d}-\mathbf{d}^{0}\right)\right)-\log \operatorname{det}\left(\widehat{\mathbf{G}}\left(\mathbf{d}^{0}\right)\right) \\
= & \log \operatorname{det}\left(\frac{1}{n} \sum_{j=j_{0}}^{j_{1}} \boldsymbol{\Lambda}_{j-<\mathcal{J}>}\left(\mathbf{d}-\mathbf{d}^{0}\right)^{-1} \boldsymbol{\Lambda}_{j}\left(\mathbf{d}^{0}\right)^{-1} I(j) \boldsymbol{\Lambda}_{j}\left(\mathbf{d}^{0}\right)^{-1} \boldsymbol{\Lambda}_{j-<\mathcal{J}>}\left(\mathbf{d}-\mathbf{d}^{0}\right)^{-1}\right) \\
& -\log \operatorname{det}\left(\widehat{\mathbf{G}}\left(\mathbf{d}^{0}\right)\right) .
\end{aligned}
$$

Define $\lambda_{j}(\delta)=2^{-(j-<\mathcal{J}>) \delta}$ for any $j \geqslant 0$ and $\delta \in \mathbb{R}$.

Let us first recall Oppenheim's inequality (see e.g. page 480 of Horn and Johnson (1990)).

Proposition 11 (Oppenheim's inequality). Let $\mathbf{E}$ and $\mathbf{B}$ be two semi-definite positive matrices. Then $\operatorname{det}(\mathbf{E} \circ \mathbf{B}) \geqslant \operatorname{det}(\mathbf{E}) \prod_{\ell} B_{\ell, \ell}$.

Let $\mathbf{A}$ be the following matrix,

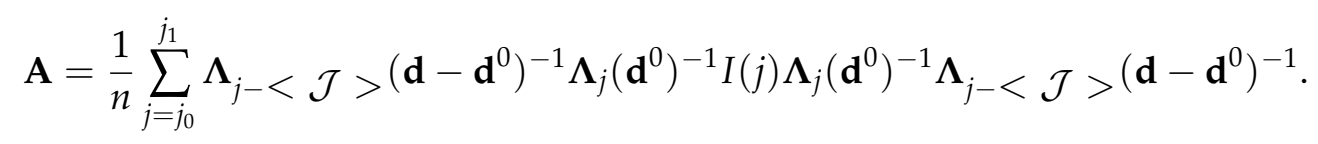

Oppenheim's inequality will be applied to matrices $\mathbf{B}$ and $\mathbf{E}\left(\mathbf{d}-\mathbf{d}^{0}\right)$ where the $(\ell, m)$-th element of $\mathbf{B}$ is defined by $B_{\ell, m}=\frac{1}{n} \sum_{j=j_{0}}^{j_{1}} n_{j} \lambda_{j}\left(d_{\ell}-d_{\ell}^{0}\right) \lambda_{j}\left(d_{m}-d_{m}^{0}\right)$ and $\mathbf{E}\left(\mathbf{d}-\mathbf{d}^{0}\right)=\mathbf{A} \circ \widetilde{\mathbf{B}}$ where $\widetilde{B}_{\ell, m}=B_{\ell, m}^{-1}$. The relation $\mathbf{A}=\mathbf{E}\left(\mathbf{d}-\mathbf{d}^{0}\right) \circ \mathbf{B}$ holds. The $(\ell, m)$-th element of $\mathbf{E}\left(\mathbf{d}-\mathbf{d}^{0}\right)$ is equal to

$$
E_{\ell, m}(\mathbf{d})=\sum_{j=j_{0}}^{j_{1}} \mu_{j, \ell, m}\left(\mathbf{d}-\mathbf{d}^{0}\right) I_{\ell, m}(j) 2^{-j\left(d_{\ell}^{0}+d_{m}^{0}\right)}
$$

$$
\text { with } \mu_{j, \ell, m}(\delta)=\frac{2^{-j\left(\delta_{\ell}+\delta_{m}\right)} 2<\mathcal{J}>\left(\delta_{\ell}+\delta_{m}\right)}{\sum_{a=j_{0}}^{j_{1}} n_{a} 2^{-a\left(\delta_{\ell}+\delta_{m}\right)} 2^{<\mathcal{J}>\left(\delta_{\ell}+\delta_{m}\right)}}=\frac{2^{-j\left(\delta_{\ell}+\delta_{m}\right)}}{\sum_{a=j_{0}}^{j_{1}} n_{a} 2^{-a\left(\delta_{\ell}+\delta_{m}\right)}} \text {. }
$$


- The matrix $\mathbf{E}$ can be expressed as $\mathbf{E}=\sum_{j, k} \widetilde{W}_{j, k} \widetilde{W}_{j, k}$. Consequently $\mathbf{E}$ is positive semi-definite being the sum of positive semi-definite matrices.

- The matrix B satisfies $\mathbf{B}=\sum_{j=j_{0}}^{j_{1}} \mathbf{M}_{j} \mathbf{M}_{j}$ with $\mathbf{M}_{j}=\left(\frac{n_{j}}{n}\right)^{1 / 2} \boldsymbol{\Lambda}_{j-<\mathcal{J}}>\left(\mathbf{d}-\mathbf{d}^{0}\right)^{-1}$. Thus $\mathbf{B}$ is also positive semi-definite.

Oppenheim's inequality implies $\log \operatorname{det}(\mathbf{A}) \geqslant \log \operatorname{det}\left(\mathbf{E}\left(\mathbf{d}-\mathbf{d}^{0}\right)\right)+\sum_{\ell} \log B_{\ell, \ell}$.

Define $L\left(\mathbf{d}-\mathbf{d}^{0}\right):=\sum_{\ell=1}^{p} \log B_{\ell, \ell}$. As we have

$$
\sum_{j=j_{0}}^{j_{1}} n_{j} \lambda_{j}(\delta) \lambda_{j}(\delta)=\sum_{j} n_{j} 2^{-2 j \delta} 2^{2<\mathcal{J}>\delta}=2^{2<\mathcal{J}>\delta} \sum_{j=j_{0}}^{j_{1}} n_{j} 2^{-2 j \delta},
$$

the function $L$ satisfies the following equality,

$$
L\left(\mathbf{d}-\mathbf{d}^{0}\right)=\sum_{\ell=1}^{p}\left[\log \left(2^{2<\mathcal{J}>\left(d_{\ell}-d_{\ell}^{0}\right)}\right)+\log \left(\frac{1}{n} \sum_{j=j_{0}}^{j_{1}} n_{j} 2^{-2 j\left(d_{\ell}-d_{\ell}^{0}\right)}\right)\right] .
$$

It is easily seen that each term of the sum corresponds to the criterion defined in Proposition 6 of Moulines et al (2008).

Inequality (15) follows with $\Delta\left(\mathbf{d}, \mathbf{d}^{0}\right)=\log \operatorname{det}\left(\mathbf{E}\left(\mathbf{d}-\mathbf{d}^{0}\right)\right)-\log \operatorname{det}\left(\widehat{\mathbf{G}}\left(\mathbf{d}^{0}\right)\right)$. We will now control the two terms in the right-hand side inequality (15).

Control of $L . L\left(\mathbf{d}-\mathbf{d}^{0}\right)$ is a multivariate extension of the criterion studied in Proposition 6 of Moulines et al (2008). It is convex, positive and minimal at $\mathbf{d}=\mathbf{d}^{0}$.

Control of $\Delta$. We shall prove that both $\log \operatorname{det} \mathbf{E}\left(\mathbf{d}-\mathbf{d}^{0}\right)$ and $\log \operatorname{det}\left(\widehat{\mathbf{G}}\left(\mathbf{d}^{0}\right)\right)$ tend uniformly to $\log \operatorname{det}\left(\mathbf{G}^{0}\right)$ for $\mathbf{d} \in \mathbb{R}^{p}$.

- The $(\ell, m)$-th element of the matrix $\mathbf{E}\left(\mathbf{d}-\mathbf{d}^{0}\right)$ is equal to

$$
E_{\ell, m}\left(\mathbf{d}-\mathbf{d}^{0}\right)=\sum_{j=j_{0}}^{j_{1}} \mu_{j, \ell, m}\left(\mathbf{d}-\mathbf{d}^{0}\right) I_{\ell, m}(j) 2^{-j\left(d_{\ell}^{0}+d_{m}^{0}\right)} \text { where } \mu_{j, \ell, m}(\delta)=\frac{2^{-j\left(\delta_{\ell}+\delta_{m}\right)}}{\sum_{a} n_{a} 2^{-a\left(\delta_{\ell}+\delta_{m}\right)}} .
$$

As $\sum_{j=j_{0}}^{j_{1}} n_{j} \mu_{j, \ell, m}(\delta)=1$, the quantity $E_{\ell, m}\left(\mathbf{d}-\mathbf{d}^{0}\right)$ is written as

$$
E_{\ell, m}\left(\mathbf{d}-\mathbf{d}^{0}\right)=G_{\ell, m}^{0}+\sum_{j, k} \mu_{j, \ell, m}\left(\mathbf{d}-\mathbf{d}^{0}\right)\left(\frac{W_{j, k}(\ell) W_{j, k}(m)}{2^{j\left(d_{\ell}^{0}+d_{m}^{0}\right)}}-G_{\ell, m}^{0}\right)
$$

where $G_{\ell, m}^{0}=\Omega_{\ell, m} K\left(d_{\ell}+d_{m}\right) \cos \left(\pi\left(d_{\ell}^{0}-d_{m}^{0}\right) / 2\right)$.

Above expression is equal to $E_{\ell, m}\left(\mathbf{d}-\mathbf{d}^{0}\right)=G_{\ell, m}^{0}+S_{\ell, m}\left(\mu_{\ell, m}\left(\mathbf{d}-\mathbf{d}^{0}\right)\right)$ with $S_{\ell, m}(\mu)$ defined previously in equation (12). Since $\sup _{\mathbf{d}}\left|\mu_{j}\left(\mathbf{d}-\mathbf{d}^{0}\right)\right| \leqslant \frac{1}{n_{j}}$, Proposition 8 states that $E_{\ell, m}\left(\mathbf{d}-\mathbf{d}^{0}\right) \rightarrow G_{\ell, m}^{0}$ uniformly in $\mathbf{d}$ when $2^{-j_{0} \beta}+N^{-1 / 2} 2^{j_{1} / 2} \rightarrow 0$. 
- Finally we shall establish that $\log \operatorname{det} \widehat{\mathbf{G}}\left(\mathbf{d}^{0}\right)$ tends to $\log \operatorname{det}\left(\mathbf{G}^{0}\right)$. Recall

$$
\widehat{G}_{\ell, m}\left(\mathbf{d}^{0}\right)=G_{\ell, m}^{0}+S_{\ell, m}(v) \text { where } v_{j}=\frac{1}{n} .
$$

The sequence $v$ belongs to the set $\mathcal{S}(0,0,1)$. Applying Proposition 9, the convergence is proved when $2^{-j_{0} \beta}+N^{-1 / 2} 2^{j_{0} / 2} \rightarrow 0$.

The consistency has been established in Proposition 10 under the condition $2^{-j_{0} \beta}+N^{-1 / 2} 2^{j_{1} / 2} \rightarrow 0$. The objective is to weaken this condition in order to prove Theorem 5 . The scheme of the proof is a generalization of the proof of Proposition 9 of Moulines et al (2008) to multivariate cases.

The only step in the proof of Proposition 10 that needs the assumption $2^{-j_{0} \beta}+N^{-1 / 2} 2^{j_{1} / 2} \rightarrow 0$ is the convergence study of $E_{\ell, m}\left(\mathbf{d}-\mathbf{d}^{0}\right)$ to $G_{\ell, m}^{0}$. The proof of Theorem 5 consists in proving that $\widehat{\mathbf{d}}>\mathbf{d}^{0}-1 / 2$ in probability in order to obtain a weaker convergence assumption for $E_{\ell, m}\left(\mathbf{d}-\mathbf{d}^{0}\right)$ applying Proposition 9.

\section{D.2 Lower bound of the estimate}

We proceed to show first that there exists $\mathbf{d}^{\text {min }}$ such that for all $\ell=1, \ldots, p$, we have $d_{\ell}^{0}-1 / 2<$ $d_{\ell}^{\text {min }}<d_{\ell}^{0}$ and $\mathbb{P}\left(\inf _{j_{1} \geqslant j_{0}+2} \widehat{d}_{\ell} \leqslant d_{\ell}^{\text {min }}\right)$ tends to 0 when $N$ goes to infinity. The proof is recursive.

STEP 1.

We introduce $\widetilde{\alpha}$ defined by

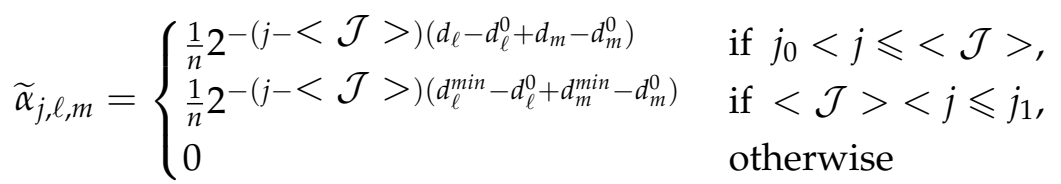

where the vector $\mathbf{d}^{\text {min }}$ is taken such that

$$
\forall \ell=1, \ldots, p, d_{\ell}^{0}-1 / 2<d_{\ell}^{\min }<d_{\ell}^{0} \text { and } \liminf _{n \rightarrow \infty} \inf _{\left\{\mathbf{d}, d_{\ell} \leqslant d_{\ell}^{\min }\right\}} \inf _{j_{1}=j_{0}, \ldots, j_{N}} \sum_{j=j_{0}}^{j_{1}} n_{j} \widetilde{\alpha}_{j, \ell, \ell}>1
$$

Let $\ell$ be a given index in $\{1, \ldots, p\}$. Following similar arguments as in Moulines et al (2008) when showing their formula (59), one can find $d_{\ell}^{\min }$ satisfying condition (16).

The quantity $R(\mathbf{d})-R\left(\mathbf{d}^{0}\right)$ is equal to

$$
\begin{aligned}
& R(\mathbf{d})-R\left(\mathbf{d}^{0}\right)=\log \operatorname{det} \mathbf{A}\left(\alpha\left(\mathbf{d}-\mathbf{d}^{0}\right)\right)-\log \operatorname{det} \widehat{\mathbf{G}}\left(\mathbf{d}^{0}\right) \\
& \text { with } A_{\ell, m}(\alpha)=\sum_{j=j_{0}}^{j_{1}} \alpha_{j, \ell, m} I_{\ell, m}(j) \text { and } \alpha_{j, \ell, m}(\delta)=\frac{1}{n} 2^{-(j-<\mathcal{J}>)\left(\delta_{\ell}+\delta_{m}\right)} .
\end{aligned}
$$


We first want to establish that for all $\mathbf{d}$ in $\left\{\mathbf{d}, \forall \ell d_{\ell} \leqslant d_{\ell}^{\min }\right\}$ we have $R(\mathbf{d})-R\left(\mathbf{d}^{0}\right) \geqslant \log \operatorname{det} \mathbf{A}(\widetilde{\alpha})-$ $\log \operatorname{det} \widehat{\mathbf{G}}\left(\mathbf{d}^{0}\right)$. To this aim we will use a generalization of Corollary 7.7.4 of Horn and Johnson (1990).

Proposition 12. Let $\mathbf{A}$ and $\mathbf{B}$ be two positive semi-definite matrices of $\mathbb{C}^{p \times p}$. Suppose $\mathbf{A}-\mathbf{B}$ is positive semi-definite. Then $\operatorname{det} \mathbf{A} \geqslant \operatorname{det} \mathbf{B}$.

Proof. We distinguish two cases:

- Suppose $\operatorname{det} \mathbf{A}=0$. Then there exists a unitary vector $x \in \mathbb{R}^{p}$ such that $x^{T} \mathbf{A} x=0$. Using the fact that $\mathbf{A}-\mathbf{B}$ is positive semi-definite, we have $x^{T}(\mathbf{A}-\mathbf{B}) x=-x^{T} \mathbf{B} x \geqslant 0$ which implies $x^{T} \mathbf{B} x=0$ since $\mathbf{B}$ is positive semi-definite. Thus $\operatorname{det} \mathbf{B}=0=\operatorname{det} \mathbf{A}$.

- Suppose $\operatorname{det} \mathbf{A}>0$. If $\operatorname{det} \mathbf{B}=0$, inequality $\operatorname{det} \mathbf{B} \leqslant \operatorname{det} \mathbf{A}$ holds. If $\operatorname{det} \mathbf{B}>0$, since $\mathbf{A}-\mathbf{B}$ is positive semi-definite we apply Corollary 7.7.4 of Horn and Johnson (1990) which concludes the proof.

Moreover to prove the positive semi-definiteness of the matrices we will use the Schur product theorem (see e.g. page 458 of Horn and Johnson (1990)).

Proposition 13 (Schur product theorem). Let $\mathbf{B}_{1}$ and $\mathbf{B}_{2}$ be two positive semi-definite matrices of $\mathbb{C}^{p \times p}$, then $\mathbf{B}_{1} \circ \mathbf{B}_{2}$ is also positive semi-definite.

Let $j \geqslant 0$. The matrix $\mathbf{B}_{1}(j)=\left(2^{j\left(d_{\ell}^{0}+d_{m}^{0}\right)} I_{\ell, m}(j)\right)_{\ell, m}$ is positive semi-definite since it can be written as $\widetilde{W}(j)^{T} \widetilde{W}(j)$. The matrix $\mathbf{B}_{2}=\left(\alpha_{j, \ell, m}\left(\mathbf{d}-\mathbf{d}^{0}\right)-\widetilde{\alpha}_{j, \ell, m}\right)_{\ell, m}$ has positive terms for all $\mathbf{d} \in\left\{\mathbf{d}, \forall \ell d_{\ell} \leqslant d_{\ell}^{\min }\right\}$ and it is thus positive semi-definite. Applying Proposition 13, we obtain that $\mathbf{B}(j)=\mathbf{B}_{1}(j) \circ \mathbf{B}_{2}(j)$ is positive semi-definite. $\mathbf{A}\left(\alpha\left(\mathbf{d}-\mathbf{d}^{0}\right)\right)-\mathbf{A}(\widetilde{\alpha})$ is then positive semi-definite being the sum of positive semi-definite matrices. Similarly, it is easy to check that both $\mathbf{A}(\widetilde{\alpha})$ and $\mathbf{A}\left(\alpha\left(\mathbf{d}-\mathbf{d}^{0}\right)\right)$ are positive semi-definite. Consequently Proposition 12 gives $\log \operatorname{det} \mathbf{A}\left(\alpha\left(\mathbf{d}-\mathbf{d}^{0}\right)\right) \geqslant$ $\log \operatorname{det} \mathbf{A}(\widetilde{\alpha})$. This result holds for all $\mathbf{d}$ satisfying $\forall \ell, d_{\ell} \leqslant d_{\ell}^{\min }$.

We now study the behaviour of $\log \operatorname{det} \mathbf{A}(\widetilde{\alpha})-\log \operatorname{det} \widehat{\mathbf{G}}\left(\mathbf{d}^{0}\right)$. First, we have proved previously (see end of section D.1) that $\log \operatorname{det} \widehat{\mathbf{G}}\left(\mathbf{d}^{0}\right)$ tends uniformly in $\mathbf{d}$ to $\log \operatorname{det} \mathbf{G}^{0}$. Second, we decompose $\mathbf{A}(\widetilde{\alpha})$ in $\mathbf{A}(\widetilde{\alpha})=\widetilde{\mathbf{G}}+\mathbf{S}(\widetilde{\alpha})$ with the elements of $\mathbf{S}(\widetilde{\alpha})$ defined in equation (12) and $\widetilde{\mathbf{G}}_{\ell, m}=\sum_{j} n_{j} \widetilde{\alpha}_{j, \ell, m} G_{\ell, m}^{0}$. We distinguish the study of the two terms.

- As $\langle\mathcal{J}\rangle \sim j_{0}$, for sufficiently large $N$ there exists a positive constant $c$ such that

$$
\left|\widetilde{\alpha}_{j, \ell, m}\right| \leqslant \frac{c}{n} 2^{\left(j-j_{0}\right)\left(d_{\ell}^{0}-d_{\ell}^{m i n}+d_{m}^{0}-d_{m}^{m i n}\right)} .
$$

Consequently, for all $(\ell, m)$, the sequence $\widetilde{\alpha}_{\ell, m}$ belongs to $\mathcal{S}(0, \gamma, c)$ with $\gamma=2 \sup _{a}\left(d_{a}^{0}-d_{a}^{\text {min }}\right)$. As the vector $\mathbf{d}^{\text {min }}$ satisfies that for any $\ell=1, \ldots, p$, we have $d_{\ell}^{0}-1 / 2<d_{\ell}^{\min } \leqslant d_{\ell}^{0}$, then $0 \leqslant \gamma<1 / 2$. Applying Proposition 9 we deduce that $\mathbf{S}(\widetilde{\alpha})$ tends to 0 in probability uniformly in $\mathbf{d}$. 
- As $\mathrm{G}^{0}$ is a covariance matrix, it is positive semi-definite. We can apply Oppenheim's inequality (Proposition 11), $\log \operatorname{det} \widetilde{\mathbf{G}} \geqslant \log \operatorname{det}\left(\mathbf{G}^{0}\right)+\sum_{\ell} \log \left(\sum_{j=j_{0}}^{j_{1}} n_{j} \widetilde{\alpha}_{j, \ell, \ell}\right)$. As we defined $\mathbf{d}^{\text {min }}$ such that (16) holds, it follows that $\lim \inf _{N \rightarrow \infty} \inf _{\left\{\mathbf{d}, \forall \ell d_{\ell} \leqslant d_{\ell}^{\min \}}\right.} \inf _{j_{1}=j_{0}, \ldots, j_{N}} \log \operatorname{det} \mathbf{A}(\widetilde{\alpha})-$ $\log \operatorname{det} \mathrm{G}^{0}>0$.

We thus get

$$
\lim _{N \rightarrow \infty} \mathbb{P}\left(\inf _{\left\{\mathbf{d}, \forall \ell d_{\ell} \leqslant d_{\ell}^{\min }\right\}} \inf _{j_{1}=j_{0}, \ldots, j_{N}} \log \operatorname{det} \mathbf{A}(\widetilde{\alpha})-\log \operatorname{det} \widehat{\mathbf{G}}\left(\mathbf{d}^{0}\right)>0\right)=1 .
$$

Suppose $\widehat{d} \in\left\{\mathbf{d}, \forall \ell d_{\ell} \leqslant d_{\ell}^{\min }\right\}$. By definition of $\widehat{\mathbf{d}}$, inequality $\inf _{\left\{\mathbf{d}, \forall m d_{m} \leqslant d_{m}^{\min }\right\}} R(d)-R\left(d^{0}\right) \leqslant 0$ holds, which is in contradiction with result (17). Finally with a probability tending to 1 there exists $\ell_{1} \in\{1, \ldots, p\}$ such that $d_{\ell_{1}} \geqslant d_{\ell_{1}}^{\text {min }}$.

STEP 2. Suppose that exist $\ell_{1}, \ell_{2}, \ldots \ell_{k}$ with $k<p$ such that, with a probability tending to 1 , $\widehat{d}_{\ell_{i}} \geqslant d_{\ell_{i}}^{\min }$ for all $i=1, \ldots, k$. We introduce $\widetilde{\alpha}^{(k)}$ defined by $\widetilde{\alpha}_{j, \ell, m}^{(k)}=\alpha_{j, \ell, m}\left(\mathbf{d}-\mathbf{d}^{0}\right)$ if $j_{0} \leqslant j \leqslant<\mathcal{J}>$,

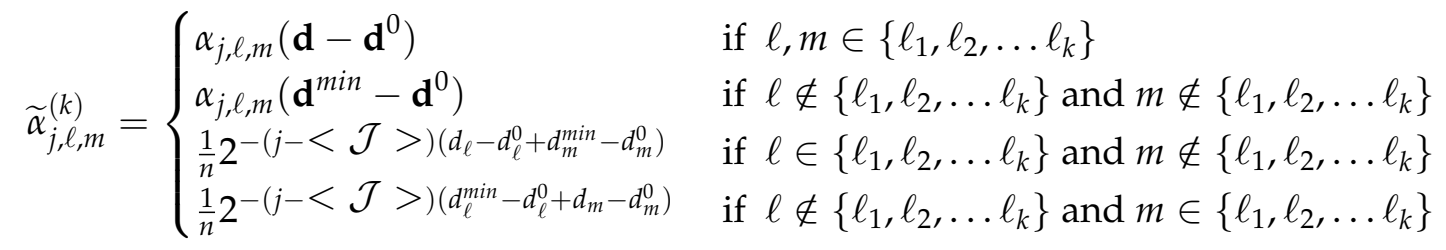

if $<\mathcal{J}><j \leqslant j_{1}$ and $\widetilde{\alpha}_{j, \ell, m}^{(k)}=0$ else. It is straightforward that for such $\widetilde{\alpha}^{(k)}$ the three following points hold,

1. For all $\mathbf{d} \in\left\{\mathbf{d}, \forall \ell \notin\left\{\ell_{1}, \ldots, \ell_{k}\right\} d_{\ell} \leqslant d_{\ell}^{\text {min }}\right\}$, for all $j \geqslant 0$, for all $(\ell, m) \in\{1, \ldots, p\}^{2}$, we have $\alpha_{j, \ell, m}\left(\mathbf{d}-\mathbf{d}^{0}\right)-\widetilde{\alpha}_{j, \ell, m}^{(k)} \geqslant 0$;

2. For all $(\ell, m) \in\{1, \ldots, p\}^{2}$, the sequence $\left(\widetilde{\alpha}_{j, \ell, m}^{(k)}\right)_{j \geqslant 0}$ belongs to $\mathcal{S}(0, \gamma, c)$ with $0 \leqslant \gamma<1 / 2$;

3. $\liminf \inf _{n \rightarrow \infty} \inf _{\left\{\mathbf{d}, \forall \ell \notin\left\{\ell_{1}, \ldots, \ell_{k}\right\} d_{\ell} \leqslant d_{\ell}^{\min }\right\}} \inf _{j_{1}=j_{0}, \ldots, j_{N}} \sum_{\ell} \log \left(\sum_{j=j_{0}}^{j_{1}} n_{j} \widetilde{\alpha}_{j, \ell_{0}, \ell_{0}}^{(k)}\right)>0$.

Analysis similar to STEP 1 shows that there exists $\ell_{k+1} \notin\left\{\ell_{1}, \ell_{2}, \ldots \ell_{k}\right\}$ such that, with a probability tending to $1, d_{\ell_{k+1}} \geqslant d_{\ell_{k+1}}^{\text {min }}$.

Step 1 and Step 2 imply that $\mathbb{P}\left(\widehat{\mathbf{d}} \in\left\{\mathbf{d}, \forall \ell=1, \ldots, p, d_{\ell} \geqslant d_{\ell}^{\min }\right\}\right) \rightarrow 1$ when $N \rightarrow \infty$.

\section{D.3 Proof of Theorem 5}

Suppose first that for sufficiently large $N$ we have $j_{1} \geqslant j_{0}+2$. 
We have established that there exists $\mathbf{d}^{\text {min }}$ such that for all $\ell=1, \ldots, p, d_{\ell}^{0}-1 / 2<d_{\ell}^{\text {min }}<d_{\ell}^{0}$ and $\mathbb{P}\left(\sup _{j_{1} \geqslant j_{0}+2} \widehat{d}_{\ell} \leqslant d_{\ell}^{\text {min }}\right)$ tends to 0 when $N$ goes to infinity.

Let $\mathbf{d}$ be a $\mathbb{R}^{p}$-vector satisfying $d_{\ell} \geqslant d_{\ell}^{\min }$ for all $\ell=1, \ldots, p$. Recall that

$$
E_{\ell, m}(\mathbf{d})=G_{\ell, m}^{0}+S_{\ell, m}\left(\mu_{\ell, m}\left(\mathbf{d}-\mathbf{d}^{0}\right)\right) \text { whith } \mu_{j, \ell, m}(\delta)=\frac{2^{-j\left(\delta_{\ell}+\delta_{m}\right)}}{\sum_{j^{\prime} \geqslant j_{0}} n_{j^{\prime}} 2^{-j^{\prime}\left(\delta_{\ell}+\delta_{m}\right)}} .
$$

We bound the sequence $\mu$ as follows:

$$
\left|\mu_{j, \ell, m}\left(\mathbf{d}-\mathbf{d}^{0}\right)\right| \leqslant \frac{2^{\left(j-j_{0}\right)\left(d_{\ell}^{0}-d_{\ell}^{m i n}+d_{m}^{0}-d_{m}^{m i n}\right)}}{\sum_{j^{\prime}=j_{0}}^{j_{1}} n_{j^{\prime}} 2^{\left(j^{\prime}-j_{0}\right)\left(d_{\ell}^{0}-d_{\ell}+d_{m}^{0}-d_{m}\right)}} \leqslant n_{j_{0}}^{-1} 2^{\left(j-j_{0}\right)\left(d_{\ell}^{0}-d_{\ell}^{m i n}+d_{m}^{0}-d_{m}^{m i n}\right)} .
$$

Since $n_{j_{0}} \sim N 2^{-j_{0}}$ and $n \sim N 2^{-j_{0}}\left(2-2^{j_{1}-j_{0}}\right)$ there exist $N$ sufficiently large such that $n_{j_{0}}^{-1} \leqslant 2 n^{-1}$. We introduce $\gamma=\sup _{\ell=1, \ldots, p} d_{\ell}^{0}-d_{\ell}^{\min }$. Then for all $(\ell, m), \mu_{\ell, m}\left(\mathbf{d}-\mathbf{d}^{0}\right)$ belongs to $\mathcal{S}(0,2 \gamma, 2)$. We have $0 \leqslant \gamma<1 / 2$ with a probability tending to 1 . Applying Proposition 9 we deduce that $E_{\ell, m}\left(\mathbf{d}-\mathbf{d}^{0}\right) \rightarrow G_{\ell, m}^{0}$ uniformly in $\mathbf{d}$ with a probability tending to 1 . Following the proof of Proposition 10 with this result, we obtain Theorem 5.

It remains to consider the case where we do not have $j_{1} \geqslant j_{0}+2$. Since we supposed $j_{1}-j_{0}$ is an increasing sequence of $N$, Proposition 10 holds, which concludes the proof.

\section{E Rate of convergence}

In order to obtain the optimal rate of convergence, we first prove the convergence with a suboptimal rate. Based on this result, we are able to obtain feasible conditions under which the rates of Theorem 6 hold.

\section{E.1 Convergence with a suboptimal rate}

We shall now establish a first rate of convergence which is not optimal but which will be useful to derive conditions for the optimal rate.

Proposition 14. Assume that (W1)-(W5) and Condition (C) hold. If in addition $j_{0}$ is chosen such that $2^{-j_{0} \beta}+N^{-1 / 2} 2^{j_{0} / 2} \rightarrow 0$ then

$$
\widehat{\mathbf{d}}-\mathbf{d}^{0}=O_{\mathbb{P}}\left(N^{-1 / 4} 2^{j_{0} / 4}+2^{-\beta j_{0} / 2}\right) .
$$

Proof. The proof is based on inequality (15). The procedure is to find a lower bound for $R(\mathbf{d})-$ $R\left(\mathbf{d}^{0}\right)$ on the set $\left\{\mathbf{d}, \max _{\ell=1, \ldots, p}\left|d_{\ell}-d_{\ell}^{0}\right|<1 / 4\right\}$. By Theorem $5, \widehat{\mathbf{d}}-\mathbf{d}^{0}$ goes to 0 in probability. Therefore, for sufficiently large $N$, we have $\max _{\ell=1, \ldots, p}\left|\widehat{d}_{\ell}-d_{\ell}^{0}\right|<1 / 4$. 
First, a second order Taylor expansion of $L\left(\mathbf{d}-\mathbf{d}^{0}\right)$ at the neighbourhood of 0 for $\mathbf{d}^{0}-1 / 4<\mathbf{d} \leqslant \mathbf{d}^{0}$ is $L\left(\mathbf{d}-\mathbf{d}^{0}\right)=\left.\frac{d^{2} L(u)}{d u^{2}}\right|_{\bar{u}}\left(\mathbf{d}-\mathbf{d}^{0}\right)^{2}+o\left(\max _{\ell=1, \ldots, p}\left|d_{\ell}-d_{\ell}^{0}\right|^{2}\right)$ with $|\bar{u}| \leqslant 1 / 4$. Proposition 6 of Moulines et al (2008) states that $\left.\liminf _{N \rightarrow \infty} \inf _{\bar{u} \in[-1 / 2,0]} \inf _{j_{1}=j_{0}+1, \ldots, j_{N}} \frac{d^{2} L(u)}{d u^{2}}\right|_{\bar{u}}>0$. Thus for $\mathbf{d}^{0}-1 / 4<\mathbf{d} \leqslant \mathbf{d}^{0}+1 / 4$, there exists $c>0$ such that $L\left(\mathbf{d}-\mathbf{d}^{0}\right)>c \sum_{\ell=1}^{p}\left(d_{\ell}-d_{\ell}^{0}\right)^{2}+$ $o\left(\max _{\ell=1, \ldots, p}\left|d_{\ell}-d_{\ell}^{0}\right|^{2}\right)$.

We now want to establish an upper bound for $\Delta\left(\mathbf{d}, \mathbf{d}^{0}\right)$. This quantity satisfies

$$
\left.\Delta\left(\mathbf{d}, \mathbf{d}^{0}\right)=\log \operatorname{det}\left(I+\mathbf{G}^{0-1} \mathbf{S}\left(\mu\left(\mathbf{d}-\mathbf{d}^{0}\right)\right)\right)\right)
$$

where $\mathbf{S}\left(\mu\left(\mathbf{d}-\mathbf{d}^{0}\right)\right)$ is the matrix with $(\ell, m)$-th element $S_{\ell, m}\left(\mu\left(\mathbf{d}-\mathbf{d}^{0}\right)\right)$ defined in (12) and $\mu_{j, \ell, m}(\cdot)$ defined in (18). Thus, $\Delta\left(\mathbf{d}, \mathbf{d}^{0}\right)=\log \left(\prod_{i=1}^{p} \lambda_{i}\right)$ where $\left(\lambda_{i}\right)_{i=1, \ldots, p}$ denote the eigenvalues of the semidefinite positive matrix $\mathbf{G}^{0-1} \mathbf{S}\left(\mu\left(\mathbf{d}-\mathbf{d}^{0}\right)\right)$. Hence

$$
0 \leqslant \Delta\left(\mathbf{d}, \mathbf{d}^{0}\right) \leqslant \operatorname{trace}\left(\mathbf{G}^{0-1} \mathbf{S}\left(\mu\left(\mathbf{d}-\mathbf{d}^{0}\right)\right)\right) .
$$

Since $\max _{\ell=1, \ldots, p}\left|d_{\ell}-d_{\ell}^{0}\right| \rightarrow 0$, for $N$ sufficiently large the quantity $\gamma=\max _{\ell=1, \ldots, p}\left|d_{\ell}-d_{\ell}^{0}\right|$ satisfies $0 \leqslant \gamma<1 / 4$. As established previously, for all $(\ell, m), \mu_{\ell, m}\left(\mathbf{d}-\mathbf{d}^{0}\right)$ belongs to $\mathcal{S}(0,2 \gamma, 2)$. Then for all $(\ell, m), S_{\ell, m}\left(\mu_{\ell, m}\left(\mathbf{d}-\mathbf{d}^{0}\right)\right)=O_{\mathbb{P}}\left(2^{-j_{0} \beta}+N^{-1 / 2} 2^{j_{0} / 2}\right)$ uniformly in $\mathbf{d} \in\left[\mathbf{d}^{0}-1 / 4, \mathbf{d}^{0}\right]$ applying Proposition 9. Hence $\Delta\left(\mathbf{d}, \mathbf{d}^{0}\right)=O_{\mathbb{P}}\left(2^{-j_{0} \beta}+N^{-1 / 2} 2^{j_{0} / 2}\right)$.

Inequality (15) thus gives

$$
R(\mathbf{d})-R\left(\mathbf{d}^{0}\right) \geqslant c \sum_{\ell=1}^{p}\left(d_{\ell}-d_{\ell}^{0}\right)^{2}+o\left(\max _{\ell}\left|d_{\ell}-d_{\ell}^{0}\right|^{2}\right)+O_{\mathbb{P}}\left(2^{-j_{0} \beta}+N^{-1 / 2} 2^{j_{0} / 2}\right)
$$

with $c>0$. It follows that for all $\ell=1, \ldots, p,\left(\widehat{d_{\ell}}-d_{\ell}^{0}\right)^{2}=O_{\mathbb{P}}\left(2^{-j_{0} \beta}+N^{-1 / 2} 2^{j_{0} / 2}\right)$.

\section{E.2 Proof of Theorem 6}

The criterion $R$ is equal to $R(\mathbf{d})=\log \operatorname{det}\left(\boldsymbol{\Lambda}_{<\mathcal{J}}>(\mathbf{d}) \widehat{\mathbf{G}}(\mathbf{d}) \boldsymbol{\Lambda}_{<\mathcal{J}}(\mathbf{d})\right)-1$. It is straightforward that $\widehat{\mathbf{d}}=\operatorname{argmin} R(\mathbf{d})$ satisfies

d

$$
\begin{array}{lll}
\widehat{\mathbf{d}}=\underset{\mathbf{d}}{\operatorname{argmin}} \bar{R}(\mathbf{d}) & \text { with } & \bar{R}(\mathbf{d})=\log \operatorname{det} \overline{\mathbf{G}}(\mathbf{d}) \\
& \text { and } \overline{\boldsymbol{G}}(\mathbf{d})=\boldsymbol{\Lambda}_{<\mathcal{J}>}\left(\mathbf{d}-\mathbf{d}^{0}\right) \widehat{\mathbf{G}}(\mathbf{d}) \boldsymbol{\Lambda}_{<\mathcal{J}>}\left(\mathbf{d}-\mathbf{d}^{0}\right)
\end{array}
$$

The Taylor expansion of $\bar{R}$ at $\widehat{\mathbf{d}}$ at the neighbourhood of $\mathbf{d}^{0}$ gives

$$
R(\widehat{\mathbf{d}})-R\left(\mathbf{d}^{0}\right)=\left.\frac{\partial \bar{R}(\mathbf{d})}{\partial \mathbf{d}}\right|_{\mathbf{d}^{0}}\left(\mathbf{d}-\mathbf{d}^{0}\right)+\left.\left(\widehat{\mathbf{d}}-\mathbf{d}^{0}\right)^{T} \frac{\partial^{2} \bar{R}(\mathbf{d})}{\partial \mathbf{d} \partial \mathbf{d}^{T}}\right|_{\overline{\mathbf{d}}}\left(\widehat{\mathbf{d}}-\mathbf{d}^{0}\right)
$$

where $\overline{\mathbf{d}}$ is such that $\left\|\overline{\mathbf{d}}-\mathbf{d}^{0}\right\| \leqslant\left\|\widehat{\mathbf{d}}-\mathbf{d}^{0}\right\|$. 
The derivatives of the criterion $\bar{R}(\mathbf{d})$ are equal to

$$
\begin{aligned}
\frac{\partial \bar{R}(\mathbf{d})}{\partial d_{a}} & =\operatorname{trace}\left(\overline{\boldsymbol{G}}(\mathbf{d})^{-1} \frac{\partial \overline{\boldsymbol{G}}(\mathbf{d})}{\partial d_{a}}\right) \\
\frac{\partial^{2} \bar{R}(\mathbf{d})}{\partial d_{a} \partial d_{b}} & =-\operatorname{trace}\left(\overline{\boldsymbol{G}}(\mathbf{d})^{-1} \frac{\partial \overline{\boldsymbol{G}}(\mathbf{d})}{\partial d_{b}} \overline{\boldsymbol{G}}(\mathbf{d})^{-1} \frac{\partial \overline{\boldsymbol{G}}(\mathbf{d})}{\partial d_{a}}\right)+\operatorname{trace}\left(\overline{\boldsymbol{G}}(\mathbf{d})^{-1} \frac{\partial^{2} \overline{\boldsymbol{G}}(\mathbf{d})}{\partial d_{a} \partial d_{b}}\right)
\end{aligned}
$$

when $\bar{G}(\mathbf{d})^{-1}$ exists.

\section{E.2.1 Study of $\bar{G}$ and its derivatives}

To study the asymptotic behaviour of the derivatives of the criterion it is necessary first to study the asymptotic behaviour of $\bar{G}$ and of its derivatives.

For any $a=1, \ldots, p$, let $\mathbf{i}_{a}$ be a $p \times p$ matrix whose a-th diagonal element is one and all other elements are zero. Let $a$ and $b$ be two indexes in $1, \ldots, p$. The first derivative of $\bar{G}(\mathbf{d})$ with respect to $d_{a}, \frac{\partial \overline{\mathbf{G}}(\mathbf{d})}{\partial d_{a}}$, is equal to

$$
-\log (2) \frac{1}{n} \sum_{j=j_{0}}^{j_{1}}(j-<\mathcal{J}>) \boldsymbol{\Lambda}_{<\mathcal{J}>}\left(\mathbf{d}-\mathbf{d}^{0}\right) \boldsymbol{\Lambda}_{j}(\mathbf{d})^{-1}\left(\mathbf{i}_{a} \mathbf{I}(j)+\mathbf{I}(j) \mathbf{i}_{a}\right) \boldsymbol{\Lambda}_{j}(\mathbf{d})^{-1} \boldsymbol{\Lambda}_{<\mathcal{J}}>\left(\mathbf{d}-\mathbf{d}^{0}\right) .
$$

And the second derivative, with respect to $d_{a}$ and $d_{b}$,

$$
\begin{aligned}
\frac{\partial^{2} \overline{\boldsymbol{G}}(\mathbf{d})}{\partial d_{a} \partial d_{b}}=\log (2)^{2} \boldsymbol{\Lambda}_{<\mathcal{J}}> & \left(\mathbf{d}-\mathbf{d}^{0}\right) \frac{1}{n} \sum_{j=j_{0}}^{j_{1}}(j-<\mathcal{J}>)^{2} \boldsymbol{\Lambda}_{j}(\mathbf{d})^{-1} \\
& \left(\mathbf{i}_{b} \mathbf{i}_{a} \mathbf{I}(j)+\mathbf{I}(j) \mathbf{i}_{a} \mathbf{i}_{b}+\mathbf{i}_{b} \mathbf{I}(j) \mathbf{i}_{a}+\mathbf{i}_{a} \mathbf{I}(j) \mathbf{i}_{b}\right) \boldsymbol{\Lambda}_{j}(\mathbf{d})^{-1} \Lambda_{<\mathcal{J}>}\left(\mathbf{d}-\mathbf{d}^{0}\right)
\end{aligned}
$$

\section{Convergence of $\bar{G}(\mathbf{d})$}

Let $\ell, m$ be given indexes in $\{1, \ldots, p\}$. Any $(\ell, m)$-th element of the matrix $\bar{G}(\mathbf{d})$ satisfies

$$
\bar{G}_{\ell, m}(\mathbf{d})=G_{\ell, m}^{0} \sum_{j=j_{0}}^{j_{1}} n_{j} \omega_{j, \ell, m}^{(0)}+S_{\ell, m}\left(\omega_{\ell, m}^{(0)}\left(\mathbf{d}-\mathbf{d}^{0}\right)\right)
$$

where $\omega_{j, \ell, m}^{(0)}\left(\mathbf{d}-\mathbf{d}^{0}\right)=\frac{1}{n} 2^{-(j-<\mathcal{J}>)\left(d_{\ell}-d_{\ell}^{0}+d_{m}-d_{m}^{0}\right)}$.

Recall that $\langle\mathcal{J}\rangle=\left(j_{0}+\eta_{j_{1}-j_{0}}\right)(1+o(1))$ with $0 \leqslant \eta_{j_{1}-j_{0}} \leqslant 1$. Let $\mathbf{d}$ be a $\mathbb{R}^{p}$-vector such that for all $\ell=1, \ldots, p, \widehat{d}_{\ell} \leqslant d_{\ell} \leqslant d_{\ell}^{0}$. As $\sup _{\ell}\left|\widehat{d}_{\ell}-d_{\ell}^{0}\right|=o_{\mathbb{P}}(1)$, for any $\gamma \in(0,1 / 2)$, there exists $N_{\gamma}$ such that for any $N \geqslant N_{\gamma}, 2^{-(j-<\mathcal{J}>)\left(d_{\ell}-d_{\ell}^{0}+d_{m}-d_{m}^{0}\right)} \leqslant 2^{\gamma} 2^{\left(j-j_{0}\right) \gamma}$. For $N \geqslant N_{\gamma}$, the sequence $\omega_{\ell, m}^{(0)}\left(\widehat{\mathbf{d}}-\mathbf{d}^{0}\right)$ belongs to $\mathcal{S}\left(0, \gamma, 2^{\gamma}\right)$. Proposition 9 shows that $S_{\ell, m}\left(\omega_{\ell, m}^{(0)}\left(\mathbf{d}-\mathbf{d}^{0}\right)\right)$ tends to zero when $2^{-j_{0} \beta}+N^{-1 / 2} 2^{j_{0} / 2} \rightarrow 0$ uniformly in $\mathbf{d}$. 
Finally, we shall prove that $\sum_{j=j_{0}}^{j_{1}} n_{j} \omega_{j, \ell, m}^{(0)}\left(\mathbf{d}-\mathbf{d}^{0}\right) \rightarrow 1$. Since $\left|2^{a}-1\right| \leqslant 2^{|a|}-1 \leqslant \log (2)|a| 2^{|a|}$ for all $a \in \mathbb{R}$,

$$
\begin{aligned}
\left|\sum_{j=j_{0}}^{j_{1}} n_{j} \omega_{j, \ell, m}^{(0)}\left(\mathbf{d}-\mathbf{d}^{0}\right)-1\right| & \leqslant \frac{1}{n} \sum_{j} n_{j} \log (2)|j-<\mathcal{J}>| \max _{\ell=1, \ldots, p}\left|d_{\ell}-d_{\ell}^{0}\right| 2^{|j-<\mathcal{J}>| \max _{\ell=1, \ldots, p}\left|d_{\ell}-d_{\ell}^{0}\right|} \\
& \leqslant 2 \log (N) \max _{\ell=1, \ldots, p}\left|d_{\ell}-d_{\ell}^{0}\right| 2^{2 \log _{2}(N) \max _{\ell=1, \ldots, p}\left|d_{\ell}-d_{\ell}^{0}\right|}
\end{aligned}
$$

The last inequality is a consequence of $j_{0} \leqslant j_{1} \leqslant j_{N}=\log _{2}(N)$. Under assumption $\log (N) \max _{\ell=1, \ldots, p}\left|\widehat{d}_{\ell}-d_{\ell}^{0}\right| \rightarrow 0$, the right-hand side of the inequality goes to 0 when $N$ goes to infinity.

Consequently,

$$
\sup _{\left\{\mathbf{d},\left\|\mathbf{d}-\mathbf{d}^{0}\right\| \leqslant\left\|\widehat{\mathbf{d}}-\mathbf{d}^{0}\right\|\right\}} \bar{G}_{a, b}(\mathbf{d})=G_{a, b}^{0}\left(1+o_{\mathbb{P}}\left(\log (N) \max _{\ell}\left|d_{\ell}-d_{\ell}^{0}\right|\right)\right)+o_{\mathbb{P}}\left(2^{-j_{0} \beta}+N^{-1 / 2} 2^{j_{0} / 2}\right) .
$$

Due to Proposition 14 , it is sufficient that $\log (N)^{2}\left(2^{-j_{0} \beta}+N^{-1 / 2} 2^{j_{0} / 2}\right) \rightarrow 0$ to obtain $\sup _{\left\{\mathbf{d},\left\|\mathbf{d}-\mathbf{d}^{0}\right\| \leqslant\left\|\widehat{\mathbf{d}}-\mathbf{d}^{0}\right\|\right\}} \bar{G}_{a, b}(\mathbf{d})=G_{a, b}^{0}+o_{\mathbb{P}}(1)$. Thus for sufficiently large $N, \bar{G}(\mathbf{d})$ is invertible and $\bar{G}(\mathbf{d})^{-1}$ converges in probability to $\mathbf{G}^{0-1}$ on the set $\left\{\mathbf{d},\left\|\mathbf{d}-\mathbf{d}^{0}\right\| \leqslant\left\|\widehat{\mathbf{d}}-\mathbf{d}^{0}\right\|\right\}$.

\section{Convergence of $\left.\frac{\partial \overline{\boldsymbol{G}}(d)}{\partial d_{a}}\right|_{\mathbf{d}}$}

This section concerns the convergence in probability of $\left(\left.\frac{\partial \overline{\mathbf{G}}(\mathbf{d})}{\partial d_{a}}\right|_{\mathbf{d}}\right)_{a, b}$ which is equal to

$$
\begin{aligned}
\left(\left.\frac{\partial \bar{G}(\mathbf{d})}{\partial d_{a}}\right|_{\mathbf{d}}\right)_{a, b} & =\log (2) \frac{1}{n} \sum_{j=j_{0}}^{j_{1}}(j-<\mathcal{J}>) 2^{-j\left(d_{a}^{0}+d_{b}^{0}\right)} I_{a, b}(j) \\
& =\log (2)\left[G_{a, b}^{0} \sum_{j=j_{0}}^{j_{1}} n_{j} \omega^{(1)}\left(\mathbf{d}-\mathbf{d}^{0}\right)+S_{a, b}\left(\omega^{(1)}\left(\mathbf{d}-\mathbf{d}^{0}\right)\right)\right],
\end{aligned}
$$

where $\omega_{j}^{(1)}(\delta)=\frac{1}{n}(j-<\mathcal{J}>) 2^{-(j-<\mathcal{J}>)\left(\delta_{\ell}+\delta_{m}\right)}$

We first study the behaviour of $\sum_{j=j_{0}}^{j_{1}} n_{j} \omega^{(1)}\left(\mathbf{d}-\mathbf{d}^{0}\right)$.

$$
\begin{aligned}
\left|\sum_{j=j_{0}}^{j_{1}} n_{j} \omega^{(1)}\left(\mathbf{d}-\mathbf{d}^{0}\right)\right| & \leqslant \frac{1}{n} \sum_{j} n_{j} \log (2)|j-<\mathcal{J}>| \max _{\ell=1, \ldots, p}\left|d_{\ell}-d_{\ell}^{0}\right| 2^{|j-<\mathcal{J}>| \max _{\ell=1, \ldots, p}\left|d_{\ell}-d_{\ell}^{0}\right|} \\
& \leqslant \log (N) \max _{\ell=1, \ldots, p}\left|d_{\ell}-d_{\ell}^{0}\right| 2^{\log _{2}(N) \max _{\ell=1, \ldots, p}\left|d_{\ell}-d_{\ell}^{0}\right|}
\end{aligned}
$$

It is thus sufficient that $\log (N) \max _{\ell=1, \ldots, p}\left|\widehat{d}_{\ell}-d_{\ell}^{0}\right| \rightarrow 0$ to have $\sum_{j=j_{0}}^{j_{1}} n_{j} \omega^{(1)}\left(\mathbf{d}-\mathbf{d}^{0}\right)=o_{\mathbb{P}}(1)$. 
Let $\mathbf{d}$ be in a neighbourhood of $\mathbf{d}^{0}$ such that $\sup _{\ell}\left|d_{\ell}-d_{\ell}^{0}\right|<\gamma$ with $0 \leqslant \gamma<1 / 2$. As $\langle\mathcal{J}\rangle \sim j_{0}+\eta_{j_{1}-j_{0}}$ with $0 \leqslant \eta_{j_{1}-j_{0}} \leqslant 1$, there exists $N_{0}$ such that for any $N \geqslant N_{0}$ the sequence $\omega^{(1)}\left(\mathbf{d}-\mathbf{d}^{0}\right)$ belongs to $\mathcal{S}\left(1, \gamma, 2^{\gamma}\right)$. Thanks to Proposition 9 it comes that $S_{a, b}\left(\omega^{(1)}(\mathbf{d}-\right.$ $\left.\left.\mathbf{d}^{0}\right)\right)=O_{\mathbb{P}}\left(2^{-j_{0} \beta}+N^{-1 / 2} 2^{j_{0} / 2}\right)$ uniformly on the neighbourhood. Consequently, $\left(\left.\frac{\partial \overline{\mathbf{G}}(\mathbf{d})}{\partial d_{a}}\right|_{\mathbf{d}}\right)_{a, b}=$ $o_{\mathbb{P}}(1)$. Finally considering similarly the other terms, when $\log (N) \max _{\ell=1, \ldots, p}\left|\widehat{d}_{\ell}-d_{\ell}^{0}\right| \rightarrow 0$, the equivalence $\left.\frac{\partial \overline{\mathbf{G}}(\mathbf{d})}{\partial d_{a}}\right|_{\mathbf{d}}=o_{\mathbb{P}}(1)$ is fulfilled under assumptions of Theorem 6.

In addition, when $\mathbf{d}=\mathbf{d}^{0}$ we have $\sum_{j=j_{0}}^{j_{1}} n_{j} \omega_{j}^{(1)}(\mathbf{0})=0$. Hence, $\left(\left.\frac{\partial \overline{\boldsymbol{G}}(\mathbf{d})}{\partial d_{a}}\right|_{\mathbf{d}^{0}}\right)_{a, b}=C_{a, b}^{(1)}$ where $C_{a, b}^{(1)}=O_{\mathbb{P}}\left(2^{-j_{0} \beta}+N^{-1 / 2} 2^{j_{0} / 2}\right)$. Or more generally,

$$
\left.\frac{\partial \overline{\mathbf{G}}(\mathbf{d})}{\partial d_{a}}\right|_{\mathbf{d}^{0}}=\mathbf{i}_{a} \mathbf{C}^{(1)}+\mathbf{C}^{(1)} \mathbf{i}_{a}
$$

where each term of the matrix $\mathbf{C}^{(1)}$ is $O_{\mathbb{P}}\left(2^{-j_{0} \beta}+N^{-1 / 2} 2^{j_{0} / 2}\right)$.

Convergence of $\left.\frac{\partial^{2} \overline{\mathbf{G}}_{a, b}(\mathbf{d})}{\partial d_{a} \partial d_{b}}\right|_{\mathbf{d}}$

Let $\mathbf{d}$ be a $\mathbb{R}^{p}$-vector such that $\left|d_{\ell}-d_{\ell}^{0}\right| \leqslant\left|\widehat{d}_{\ell}-d_{\ell}^{0}\right|$ for all $\ell=1, \ldots, p$. The proof is derived for $\left(\left.\frac{\partial^{2} \overline{\boldsymbol{G}}_{a, b}(\mathbf{d})}{\partial d_{a} \partial d_{b}}\right|_{\mathbf{d}}\right)_{a, b}$, for $a \neq b$. The argumentation is similar for the diagonal terms. Introducing a sequence $S_{a, b}(\cdot)$, the expression (23) is rewritten as

$$
\begin{aligned}
\left(\left.\frac{\partial^{2} \bar{G}_{a, b}(\mathbf{d})}{\partial d_{a} \partial d_{b}}\right|_{\mathbf{d}}\right)_{a, b} & =\log (2)^{2}\left[2^{<\mathcal{J}>\left(d_{a}+d_{b}-d_{a}^{0}-d_{b}^{0}\right)} \frac{1}{n} \sum_{j=j_{0}}^{j_{1}}(j-<\mathcal{J}>)^{2} \frac{I_{a, b}(j)}{2^{j\left(d_{a}+d_{b}\right)}}\right] \\
& =\log (2)^{2}\left[G_{a, b}^{0} \sum_{j=j_{0}}^{j_{1}} n_{j} \omega_{j, a, b}^{(2)}\left(\mathbf{d}-\mathbf{d}^{0}\right)+S_{a, b}\left(\omega_{a, b}^{(2)}\left(\mathbf{d}-\mathbf{d}^{0}\right)\right)\right]
\end{aligned}
$$

where $\omega_{j, a, b}^{(2)}(\delta)=\frac{1}{n}(j-<\mathcal{J}>)^{2} 2^{-(j-<\mathcal{J}>)\left(\delta_{a}+\delta_{b}\right)}$.

First, we want to prove that

$$
\sum_{j=j_{0}}^{j_{1}} n_{j} \omega_{j, a, b}^{(2)}\left(\mathbf{d}-\mathbf{d}^{0}\right)=\kappa_{j_{1}-j_{0}}\left(1+o_{\mathbb{P}}(1)\right)
$$

Recall $\kappa_{j_{1}-j_{0}}=\frac{1}{n} \sum_{j=j_{0}}^{j_{1}}(j-<\mathcal{J}>)^{2} n_{j}$. Since $\left|2^{a}-1\right| \leqslant 2^{|a|}-1 \leqslant \log (2)|a| 2^{|a|}$ for all $a \in \mathbb{R}$, we have the inequality

$$
\left|\sum_{j=j_{0}}^{j_{1}} n_{j} \omega_{j, a, b}^{(2)}\left(\overline{\mathbf{d}}-\mathbf{d}^{0}\right)-\kappa_{j_{1}-j_{0}}\right| \leqslant 2 \log (2) \kappa_{j_{1}-j_{0}} \log _{2}(N)\left|d-d^{0}\right| 2^{2 \log _{2}(N)\left|d-d^{0}\right|} .
$$


Equation (26) holds when $\log (N) \max _{\ell=1, \ldots, p}\left|d_{\ell}-d_{\ell}^{0}\right| \rightarrow 0$. Due to Proposition 14 it is sufficient that $\log (N)^{2}\left(2^{-j_{0} \beta}+N^{-1 / 2} 2^{j_{0} / 2}\right) \rightarrow 0$.

Let $\mathbf{d}$ be in a neighbourhood of $\mathbf{d}^{0}$ such that $\sup _{\ell}\left|d_{\ell}-d_{\ell}^{0}\right|<\gamma$ with $0 \leqslant \gamma<1 / 2$. As $<\mathcal{J}>\sim$ $j_{0}+\eta_{j_{1}-j_{0}}$ with $0 \leqslant \eta_{j_{1}-j_{0}} \leqslant 1$, there exists $N_{0}$ such that for any $N \geqslant N_{0}$ the sequence $\omega_{a, b}^{(2)}\left(\mathbf{d}-\mathbf{d}^{0}\right)$ belongs to the set $\mathcal{S}\left(2, \gamma, 2^{\gamma}\right)$. Using Proposition 9, $S_{a, b}\left(\omega_{a, b}^{(2)}\left(\mathbf{d}-\mathbf{d}^{0}\right)\right) \leqslant C\left(2^{-j_{0} \beta}+N^{-1 / 2} 2^{j_{0} / 2}\right)$ uniformly on the neighbourhood of $\mathbf{d}$ for $N \geqslant N_{0}$. As a consequence,

$$
\left(\left.\frac{\partial^{2} \overline{\boldsymbol{G}}_{a, b}(\mathbf{d})}{\partial d_{a} \partial d_{b}}\right|_{\overline{\mathbf{d}}}\right)_{a, b}=\log (2)^{2} \kappa_{j_{1}-j_{0}} G_{a, b}^{0}\left(1+o_{\mathbb{P}}(1)\right)+C_{a, b}^{(2)}
$$

with $C_{a, b}^{(2)}=O_{\mathbb{P}}\left(2^{-j_{0} \beta}+N^{-1 / 2} 2^{j_{0} / 2}\right)$.

Finally, when $N$ goes to infinity,

$$
\left.\sup _{\left\{\mathbf{d},\left\|\mathbf{d}-\mathbf{d}^{0}\right\| \leqslant\left\|\widehat{\mathbf{d}}-\mathbf{d}^{0}\right\|\right\}} \frac{\partial^{2} \bar{G}_{a, b}(\mathbf{d})}{\partial d_{a} \partial d_{b}}\right|_{\overline{\mathbf{d}}}=\log (2)^{2} \kappa_{j_{1}-j_{0}}\left(\mathbf{i}_{b} \mathbf{i}_{a} \mathbf{G}^{0}+\mathbf{i}_{b} \mathbf{G}^{0} \mathbf{i}_{a}+\mathbf{i}_{a} \mathbf{G}^{0} \mathbf{i}_{b}+\mathbf{G}^{0} \mathbf{i}_{a} \mathbf{i}_{b}\right)+o_{\mathbb{P}}(1)
$$

when $\log (N) \max _{\ell=1, \ldots, p}\left|\widehat{d}_{\ell}-d_{\ell}^{0}\right|=o_{\mathbb{P}}(1)$ and $2^{-j_{0} \beta}+N^{-1 / 2} 2^{j_{0} / 2} \rightarrow 0$.

\section{E.2.2 Second derivative of the criterion}

Let us detail the expression of the second derivative of $R$ with respect to $\mathbf{d}$ at $\overline{\mathbf{d}}$,

$$
\left.\frac{\partial^{2} \bar{R}(\mathbf{d})}{\partial d_{a} \partial d_{b}}\right|_{\overline{\mathbf{d}}}=-\operatorname{trace}\left(\left.\left.\overline{\boldsymbol{G}}(\overline{\mathbf{d}})^{-1} \frac{\partial \overline{\boldsymbol{G}}(\mathbf{d})}{\partial d_{b}}\right|_{\overline{\mathbf{d}}} \overline{\boldsymbol{G}}(\overline{\mathbf{d}})^{-1} \frac{\partial \overline{\boldsymbol{G}}(\mathbf{d})}{\partial d_{a}}\right|_{\overline{\mathbf{d}}}\right)+\operatorname{trace}\left(\left.\overline{\boldsymbol{G}}(\overline{\mathbf{d}})^{-1} \frac{\partial^{2} \overline{\boldsymbol{G}}(\mathbf{d})}{\partial d_{a} \partial d_{b}}\right|_{\overline{\mathbf{d}}}\right) .
$$

Using (24) and the previous study that established that $\left.\frac{\partial \bar{G}(\mathbf{d})}{\partial d_{a}}\right|_{\overline{\mathbf{d}}}=o_{\mathbb{P}}(1)$, the first term tends to 0 under assumptions of Theorem 6. Combining (24) and (27) we can assert that $\left.\frac{\partial^{2} \bar{R}(\mathbf{d})}{\partial d_{a} \partial d_{b}}\right|_{\overline{\mathbf{d}}}$ tends in probability to

$$
\log (2)^{2} \kappa_{j_{1}-j_{0}} \operatorname{trace}\left(\mathbf{G}^{0-1}\left(\mathbf{i}_{b} \mathbf{i}_{a} \mathbf{G}^{0}+\mathbf{i}_{b} \mathbf{G}^{0} \mathbf{i}_{a}+\mathbf{i}_{a} \mathbf{G}^{0} \mathbf{i}_{b}+\mathbf{G}^{0} \mathbf{i}_{a} \mathbf{i}_{b}\right)\right) .
$$

Let $G_{\ell, m}^{0(-1)}$ denotes the $(\ell, m)$-th element of $\mathbf{G}^{0-1}$. When $a \neq b$,

$$
\operatorname{trace}\left(\mathbf{G}^{0-1}\left(\mathbf{i}_{b} \mathbf{i}_{a} \mathbf{G}^{0}+\mathbf{i}_{b} \mathbf{G}^{0} \mathbf{i}_{a}+\mathbf{i}_{a} \mathbf{G}^{0} \mathbf{i}_{b}+\mathbf{G}^{0} \mathbf{i}_{a} \mathbf{i}_{b}\right)\right)=G_{a, b}^{0(-1)} G_{a, b}^{0}+G_{b, a}^{0(-1)} G_{b, a}^{0}=2 G_{a, b}^{0(-1)} G_{a, b}^{0} .
$$

When $a=b$,

$$
\operatorname{trace}\left(\mathbf{G}^{0-1}\left(\mathbf{i}_{b} \mathbf{i}_{a} \mathbf{G}^{0}+\mathbf{i}_{b} \mathbf{G}^{0} \mathbf{i}_{a}+\mathbf{i}_{a} \mathbf{G}^{0} \mathbf{i}_{b}+\mathbf{G}^{0} \mathbf{i}_{a} \mathbf{i}_{b}\right)\right)=2\left(1+G_{a, a}^{0(-1)} G_{a, a}^{0}\right) .
$$

Finally,

$$
\left.\frac{\partial^{2} \bar{R}(\mathbf{d})}{\partial \mathbf{d} \partial \mathbf{d}^{T}}\right|_{\overline{\mathbf{d}}}=\kappa_{j_{1}-j_{0}} 2 \log (2)^{2}\left(\mathbf{G}^{0-1} \circ \mathbf{G}^{0}+\mathbf{I}_{p}\right)+o_{\mathbb{P}}(1) .
$$

The matrix $\mathrm{G}^{0-1} \circ \mathrm{G}^{0}$ is positive definite using Schur product theorem (Proposition 13) and hence the matrix $\mathrm{G}^{0-1} \circ \mathrm{G}^{0}+\mathbf{I}_{p}$ is invertible. 


\section{E.2.3 End of the proof}

The Taylor expansion (20) together with (28) imply

$$
\begin{aligned}
2 \log (2)^{2} \kappa_{j_{1}-j_{0}}\left(\widehat{\mathbf{d}}-\mathbf{d}^{0}\right) & =-\left.\left(\left.\frac{1}{2 \log (2)^{2} \kappa_{j_{1}-j_{0}}} \frac{\partial^{2} \bar{R}(\mathbf{d})}{\partial \mathbf{d} \partial \mathbf{d}^{T}}\right|_{\overline{\mathbf{d}}}\right)^{-1} \frac{\partial \bar{R}(\mathbf{d})}{\partial \mathbf{d}}\right|_{\mathbf{d}^{0}} \\
& =\left.\left(\mathbf{G}^{0-1} \circ \mathbf{G}^{0}+\mathbf{I}_{p}\right)^{-1} \frac{\partial \bar{R}(\mathbf{d})}{\partial \mathbf{d}}\right|_{\mathbf{d}^{0}}\left(1+o_{\mathbb{P}}(1)\right)
\end{aligned}
$$

We now study the convergence of $\left.\frac{\partial \bar{R}(\mathbf{d})}{\partial \mathbf{d}}\right|_{\mathbf{d}^{0}}$. Using equations (21) and (25), we have the equation

$$
\left.\frac{\partial \bar{R}(\mathbf{d})}{\partial d_{a}}\right|_{\mathbf{d}^{0}}=2 \sum_{b=1}^{p} \overline{\boldsymbol{G}}_{a, b}^{(-1)}\left(\mathbf{d}^{0}\right) C_{a, b}^{(1)} .
$$

So the asymptotic behaviour of the first derivative of the criterion is

$$
\left.\frac{\partial \bar{R}(\mathbf{d})}{\partial d_{a}}\right|_{\mathbf{d}^{0}}=O_{\mathbb{P}}\left(2^{-j_{0} \beta}+N^{-1 / 2} 2^{j_{0} / 2}\right) .
$$

Plugging this result into the expression above, it comes

$$
\kappa_{j_{1}-j_{0}}\left(2^{-j_{0} \beta}+N^{-1 / 2} 2^{j_{0} / 2}\right)^{-1}\left(\widehat{\mathbf{d}}-\mathbf{d}^{0}\right)=O_{\mathbb{P}}(1) .
$$

Since $\kappa_{\ell}>0$ for $\ell \geqslant 1$ and $\kappa_{\ell} \rightarrow 2$ when $\ell \rightarrow \infty$, the sequence $\kappa_{j_{1}-j_{0}}$ is bounded below by a positive constant. The rate of convergence for $\widehat{\mathbf{d}}-\mathbf{d}^{0}$ in Theorem 6 follows.

\section{E.3 Convergence of $\widehat{G}(\widehat{d})$ and of $\widehat{\Omega}$}

Recall $\widehat{\mathbf{G}}_{\ell, m}(\widehat{\mathbf{d}})=2^{<\mathcal{J}>\left(\widehat{d}_{\ell}-d_{\ell}^{0}+\widehat{d}_{m}-d_{m}^{0}\right)} \bar{G}_{\ell, m}(\widehat{\mathbf{d}})$. Equation (24) with the rate obtained for the convergence of $\widehat{\mathbf{d}}-\mathbf{d}^{0}$ state that $\bar{G}_{\ell, m}(\widehat{\mathbf{d}})=G_{\ell, m}^{0}\left(1+O_{\mathbb{P}}\left(\log (N)\left(2^{-j_{0} \beta}+N^{-1 / 2} 2^{j_{0} / 2}\right)\right)+\right.$ $O_{\mathbb{P}}\left(2^{-j_{0} \beta}+N^{-1 / 2} 2^{j_{0} / 2}\right)$ under assumptions of Theorem 6 . The rate of convergence of $\widehat{G}_{\ell, m}(\widehat{\mathbf{d}})$ in Theorem 6 is then derived from the fact that $2<\mathcal{J}>u-1=j_{0} u \log (2)(1+o(1))$ when $u \rightarrow 0$.

The convergence of $\widehat{\Omega}$ is straightforward, thanks to the fact that $K(\cdot)$ is a continuous function of d. To obtain the rate of convergence, we observe first that $\cos (u)=1+o\left(u^{2}\right)$ when $u$ goes to 0 . Second,

$$
\begin{aligned}
K\left(\widehat{d}_{\ell}+\widehat{d}_{m}\right)-K\left(d_{\ell}^{0}+d_{m}^{0}\right) & =\int_{-\infty}^{\infty}\left(|\lambda|^{-\widehat{d}_{\ell}-\widehat{d}_{m}}-|\lambda|^{-d_{\ell}^{0}-d_{m}^{0}}\right)|\widehat{\psi}(\lambda)|^{2} d \lambda \\
& \leqslant\left|\widehat{d}_{\ell}+\widehat{d}_{m}-d_{\ell}^{0}-d_{m}^{0}\right| \int_{-\infty}^{\infty}|\log | \lambda|||\lambda|^{-d_{\ell}^{0}-d_{m}^{0}}|\widehat{\psi}(\lambda)|^{2} d \lambda .
\end{aligned}
$$

Using assumption (W2) and (W5),

$$
\left|K\left(\widehat{d}_{\ell}+\widehat{d}_{m}\right)-K\left(d_{\ell}^{0}+d_{m}^{0}\right)\right| \leqslant\left|\widehat{d}_{\ell}+\widehat{d}_{m}-d_{\ell}^{0}-d_{m}^{0}\right| C \int_{-\infty}^{\infty}|\log | \lambda|||\lambda|^{-(1+\beta)} d \lambda
$$


with $C$ positive constant. The integral on the right-hand side is finite and thus $K\left(\widehat{d}_{\ell}+\widehat{d}_{m}\right)-K\left(d_{\ell}^{0}+\right.$ $\left.d_{m}^{0}\right)=O_{\mathbb{P}}\left(\max _{i=1, \ldots, p}\left|\widehat{d_{i}}-d_{i}^{0}\right|\right)$. When $\max _{i=1, \ldots, p}\left|\widehat{d_{i}}-d_{i}^{0}\right|=O_{\mathbb{P}}\left(2^{-j_{0} \beta}+N^{-1 / 2} 2^{j_{0} / 2}\right)$, we have $1 /\left(K\left(\widehat{d}_{\ell}+\widehat{d}_{m}\right) \cos \left(\frac{\pi}{2}\left(\widehat{d}_{\ell}-\widehat{d}_{m}\right)\right)\right)=\left(1+O_{\mathbb{P}}\left(2^{-j_{0} \beta}+N^{-1 / 2} 2^{j_{0} / 2}\right)\right)$ which concludes the proof.

\section{E.4 Additional tools}

These results correspond to Lemma 13 of Moulines et al (2008)

Define the sequences $\eta_{L}$ and $\kappa_{L}$ for any $L \geqslant 0$ by

$$
\begin{aligned}
& \eta_{L}:=\sum_{i=0}^{L} i \frac{2^{-i}}{2-2^{-L}} \\
& \kappa_{L}:=\sum_{i=0}^{L}\left(i-\eta_{L}\right)^{2} \frac{2^{-i}}{2-2^{-L}}
\end{aligned}
$$

It is straightforward that

$$
\begin{aligned}
n & \sim N 2^{-j_{0}}\left(2-2^{-\left(j_{1}-j_{0}\right)}\right) \\
<\mathcal{J}> & \sim j_{0}+\eta_{j_{1}-j_{0}} \\
\frac{1}{n} \sum_{j=j_{0}}^{j_{1}}(j-<\mathcal{J}>)^{2} n_{j} & \sim \kappa_{j_{1}-j_{0}}
\end{aligned}
$$

For every $L \geqslant 1$ the quantities $\eta_{L}$ and $\kappa_{L}$ are strictly positive. When $L$ goes to infinity, the sequences $\eta_{L}$ and $\kappa_{L}$ respectively converge to 1 and 2 .

And for all $u \geqslant 0$,

$$
\frac{1}{\kappa_{L}} \sum_{i=0}^{L-u} \frac{2^{-i}}{2-2^{-L}}\left(i-\eta_{L}\right)\left(i+u-\eta_{L}\right) \rightarrow 1 \text { when } L \rightarrow \infty
$$

\section{References}

Abadir KM, Distaso W, Giraitis L (2007) Nonstationarity-extended local Whittle estimation. Journal of Econometrics 141(2):1353-1384

Abry P, Veitch D (1998) Wavelet analysis of long-range-dependent traffic. Information Theory, IEEE Transactions on 44(1):2-15

Achard S, Bassett DS, Meyer-Lindenberg A, Bullmore E (2008) Fractal connectivity of long-memory networks. Physical Review E 77(3):036,104

Bardet J, Lang G, Moulines E, Soulier P (2000) Wavelet estimator of long-range dependent processes. Statistical Inference for Stochastic Processes 3(1-2):85-99

Chambers M (1995) The simulation of random vector time series with given spectrum. Mathematical and Computer Modelling 22(2):1-6 
Coeurjolly JF, Amblard PO, Achard S (2013) Wavelet analysis of the multivariate fractional Brownian motion. ESAIM: Probability and Statistics 17:592-604

Dahlhaus R (1989) Efficient parameter estimation for self-similar processes. The Annals of Statistics 17(4):1749-1766

Daubechies I (1992) Ten lectures on wavelets, vol 61. SIAM, Philadelphia

Didier G, Pipiras V (2011) Integral representations and properties of operator fractional Brownian motions. Bernoulli 17(1):1-33

Faÿ G, Moulines E, Roueff F, Taqqu MS (2009) Estimators of long-memory: Fourier versus wavelets. Journal of Econometrics 151(2):159-177

Fox R, Taqqu MS (1986) Large-sample properties of parameter estimates for strongly dependent stationary gaussian time series. The Annals of Statistics 14(2):517-532

Frías M, Alonso F, Ruiz-Medina M, Angulo J (2008) Semiparametric estimation of spatial long-range dependence. Journal of Statistical Planning and Inference 138(5):1479-1495

Gencay R, Signori D (2015) Multi-scale tests for serial correlation. Journal of Econometrics 184(1):62-80

Gençay R, Selçuk F, Whitcher BJ (2001) An introduction to wavelets and other filtering methods in finance and economics. Academic Press, New York

Geweke J, Porter-Hudak S (1983) The estimation and application of long memory time series models. Journal of Time Series Analysis 4(4):221-238

Giraitis L, Robinson PM, Samarov A (1997) Rate optimal semiparametric estimation of the memory parameter of the gaussian time series with long-range dependence. Journal of Time Series Analysis 18(1):49-60

Gonzaga A, Hauser M (2011) A wavelet Whittle estimator of generalized long-memory stochastic volatility. Statistical Methods \& Applications 20(1):23-48

Hannan EJ (1973) The asymptotic theory of linear time-series models. Journal of Applied Probability pp 130-145

Horn RA, Johnson CR (1990) Matrix analysis. Cambridge university press, New York

Hurvich CM, Chen WW (2000) An efficient taper for potentially overdifferenced long-memory time series. Journal of Time Series Analysis 21(2):155-180

Iouditsky A, Moulines E, Soulier P, et al (2001) Adaptive estimation of the fractional differencing coefficient. Bernoulli 7(5):699-731

Jensen MJ (1999) An approximate wavelet MLE of short and long memory parameters. Studies in Nonlinear Dynamics and Econometrics 3(4):239-253

Kechagias S, Pipiras V (2015) Definitions and representations of multivariate long-range dependent time series. Journal of Time Series Analysis 36(1):1-25, DOI 10.1111/jtsa.12086 
Kokoszka PS, Taqqu MS (1995) Fractional arima with stable innovations. Stochastic processes and their applications 60(1):19-47

Lobato IN (1997) Consistency of the averaged cross-periodogram in long memory series. Journal of Time Series Analysis 18(2):137-155

Lobato IN (1999) A semiparametric two-step estimator in a multivariate long memory model. Journal of Econometrics 90(1):129-153

Mandelbrot BB, Van Ness JW (1968) Fractional Brownian motions, fractional noises and applications. SIAM Review 10(4):422-437

Maxim V, Şendur L, Fadili MJ, Suckling J, Gould R, Howard R, Bullmore ET (2005) Fractional Gaussian noise, functional MRI and Alzheimer's disease. NeuroImage 25:141-158

Moulines E, Roueff F, Taqqu MS (2007) On the spectral density of the wavelet coefficients of longmemory time series with application to the log-regression estimation of the memory parameter. Journal of Time Series Analysis 28(2):155-187

Moulines E, Roueff F, Taqqu MS (2008) A wavelet Whittle estimator of the memory parameter of a nonstationary gaussian time series. The Annals of Statistics 36(4):1925-1956

Nielsen FS (2011) Local Whittle estimation of multi-variate fractionally integrated processes. Journal of Time Series Analysis 32(3):317-335

Nielsen MØ, Frederiksen PH (2005) Finite sample comparison of parametric, semiparametric, and wavelet estimators of fractional integration. Econometric Reviews 24(4):405-443

Papanicolaou GC, Sølna K (2003) Wavelet based estimation of local Kolmogorov turbulence. In: Doukhan P, Oppenheim G, Taqqu MS (eds) Theory and Applications of Long-range Dependence, Birkhäuser Boston, Boston, MA, pp 473-505

Percival DB, Walden AT (2006) Wavelet methods for time series analysis, vol 4. Cambridge University Press, New York

Phillips PC, Shimotsu K (2004) Local Whittle estimation in nonstationary and unit root cases. The Annals of Statistics 32(2):656-692

Robinson PM (1994a) Rates of convergence and optimal spectral bandwidth for long range dependence. Probability Theory and Related Fields 99(3):443-473

Robinson PM (1994b) Semiparametric analysis of long-memory time series. The Annals of Statistics 22(1):515-539

Robinson PM (1995a) Gaussian semiparametric estimation of long range dependence. The Annals of Statistics 23(5):1630-1661

Robinson PM (1995b) Log-periodogram regression of time series with long range dependence. The Annals of Statistics 23(3):1048-1072

Robinson PM (2005) Robust covariance matrix estimation: HAC estimates with long memory/antipersistence correction. Econometric Theory 21(01):171-180 
Sela RJ, Hurvich CM (2008) Computationaly efficient methods for two multivariate fractionnaly integrated models. Journal of Time Series Analysis 30:6, DOI 10.1111/jtsa.12086

Sela RJ, Hurvich CM (2012) The averaged periodogram estimator for a power law in coherency. Journal of Time Series Analysis 33(2):340-363

Shimotsu K (2007) Gaussian semiparametric estimation of multivariate fractionally integrated processes. Journal of Econometrics 137(2):277-310

Velasco C, Robinson PM (2000) Whittle pseudo-maximum likelihood estimation for nonstationary time series. Journal of the American Statistical Association 95(452):1229-1243

Wang L, Wang J (2014) Wavelet estimation of the memory parameter for long range dependent random fields. Statistical Papers 55(4):1145-1158

Whitcher B, Jensen MJ (2000) Wavelet estimation of a local long memory parameter. Exploration Geophysics 31(1/2):94-103

Wornell GW, Oppenheim AV (1992) Estimation of fractal signals from noisy measurements using wavelets. Signal Processing, IEEE Transactions on 40(3):611-623 